\title{
A bHLH-PAS protein regulates light-dependent diurnal rhythmic processes in the marine diatom Phaeodactylum tricornutum
}

Rossella Annunziata ${ }^{\mathrm{a}, \mathrm{b}, 1}$, Andrés Ritter ${ }^{\mathrm{a}, 1}$, Antonio Emidio Fortunato ${ }^{\mathrm{a}, \mathrm{c}, 1}$, Soizic CheminantNavarro $^{a}$, Nicolas Agier ${ }^{\mathrm{a}}$, Marie J. J. Huysman ${ }^{\mathrm{a}, \mathrm{d}, \mathrm{e}^{*}}$, Per Winge ${ }^{\mathrm{f}}$, Atle Bones ${ }^{\mathrm{f}}$, François-Yves Bouget $^{\mathrm{h}}$, Marco Cosentino Lagomarsino ${ }^{\mathrm{a}}$, Jean Pierre Bouly ${ }^{\mathrm{a}}$, and Angela Falciatore ${ }^{\mathrm{a}, 2}$.

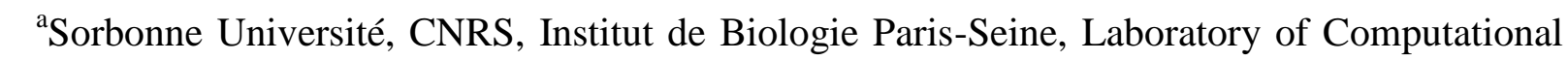
and Quantitative Biology, F-75005, Paris, France.

${ }^{\mathrm{b}}$ Stazione Zoologica Anton Dohrn, Villa Comunale, 80121 Napoli, Italy.

${ }^{\mathrm{c}}$ ImmunRise Technologies, 75006 Paris, France.

${ }^{\mathrm{d}}$ Ghent University, Department of Plant Biotechnology and Bioinformatics, Technologiepark 927, B-9052 Ghent, Belgium.

${ }^{\mathrm{e}}$ VIB Center for Plant Systems Biology, Technologiepark 927, B-9052 Ghent, Belgium.

${ }^{\mathrm{f}}$ Department of Biology, NTNU Norwegian University of Science and Technology, 7491 Trondheim, Norway.

${ }^{\mathrm{h}}$ Sorbonne Université, UMR 7621, Laboratoire d'Océanographie Microbienne, Observatoire Océanologique, Banyuls-sur-Mer, France.

${ }^{1}$ These authors contributed equally to this work.

*Disclaimer: The author (MJJH) is currently an employee of the European Research Council Executive Agency. The views expressed are purely those of the writer and may not in any circumstances be regarded as stating an official position of the European Commission.

\section{${ }^{2}$ Corresponding Author:}

Angela Falciatore

Diatom Functional Genomics Team

Laboratory of Computational and Quantitative Biology (LCQB)

Institut de Biologie Paris-Seine (IBPS)

Jussieu Campus - Sorbonne Université

4, Place Jussieu, 75005 Paris, France

Tel: +330144278142

32 Email: angela.falciatore@upmc.fr

3 Keywords: Diatoms, diurnal rhythms, transcription factors, cell division, gene expression. 


\section{ABSTRACT}

Periodic light-dark cycles govern the timing of basic biological processes in organisms inhabiting land as well as the sea, where life evolved. Although prominent marine phytoplanktonic organisms such as diatoms show robust diurnal rhythms in growth, cell cycle and gene expression, the molecular foundations controlling these processes are still obscure. By exploring the regulatory landscape of diatom diurnal rhythms, we unveil the function of a Phaeodactylum tricornutum bHLH-PAS protein, PtbHLH1a, in the regulation of lightdependent diurnal rhythms. Peak expression of PtbHLHIa mRNA occurs toward the end of the light period and it adjusts to photoperiod changes. Ectopic over-expression of PtbHLH1a results in lines showing a phase shift in diurnal cell fluorescence, compared to the wild-type cells, and with altered cell cycle progression and gene expression. Reduced oscillations in gene expression are also observed in overexpression lines compared to wild-type in continuous darkness, showing that the regulation of rhythmicity by PtbHLH1a is not directly dependent on light inputs and cell division. PtbHLH1a homologs are widespread in diatom genomes which may indicate a common function in many species. This study adds new elements to understand diatom biology and ecology and offers new perspectives to elucidate timekeeping mechanisms in marine organisms belonging to a major, but underinvestigated branch of the tree of life.

\section{SIGNIFICANCE STATEMENT}

Most organisms experience diurnal light-dark changes and show rhythms of basic biological processes such that they occur at optimal times of the day. The ocean harbours a huge diversity of organisms showing light-dependent rhythms, but their molecular foundations are still largely unknown. In this study, we discover a novel protein, PtbHLH1a that regulates cell division, gene expression and the diurnal timing of these events in the marine diatom Phaedoactylum tricornutum. The identification of PtbHLHla-like genes in many diatom species suggests a conserved function in diurnal rhythm regulation in the most species-rich group of algae in the ocean. This study unveils critical features of diatom biology and advances the field of marine rhythms and their environmental regulation. 


\section{INTRODUCTION}

The Earth's rotation means that life evolved under a $24 \mathrm{~h}$ diurnal cycle of alternate light and dark periods. Most living organisms have developed daily rhythms of many fundamental biological processes, ranging from physiology to behaviour, such that they occur at optimal times of the day (1) which can enhance fitness $(2,3)$. These rhythms are the product of the coordinated action of signals from endogenous timekeepers, together with environmental and metabolic inputs $(4,5)$. Robust diel rhythms in growth, cell cycle, gene expression, pigment synthesis, phototactic movements and bioluminescence have been also observed in a variety of phytoplanktonic organisms, including diatoms (6-13). Diatoms represent the most speciesrich group of algae in the ocean and populate a wide range of aquatic environments $(14,15)$. These algae of the Stramenopile lineage show peculiar genomic, metabolic and cellular features, and are evolutionarily distant from the most studied model organisms in the field of biological rhythms (16-20). Diatoms have an impressive capacity to deal with environmental changes thanks to sophisticated acclimation mechanisms (21-25). Recent genome-wide analyses also showed that $25 \%$ of the diurnal transcriptome is influenced by light-dark cycles in the centric diatom Thalasiossira pseudonana (26). Moreover, detailed diurnal studies in the pennate diatom Phaeodactylum tricornutum highlighted a strict temporal separation of transcriptional gene networks $(27,28)$, as previously observed in other algae (29). Tight diurnal control of the $P$. tricornutum cell cycle has also been observed $(27,30,31)$, with light onset triggering cell cycle progression through the transcriptional activation of the diatomspecific cyclin dsCYC2 by the blue light sensor Aureochrome-1a (30). Furthermore, the transcription factor bZIP14 has recently been identified as a diurnal activator of the tricarboxylic acid (TCA) cycle, a process restricted to the late afternoon in diatoms (32). Together, these studies illustrate complex regulation of diurnal cellular activities in $P$. tricornutum. However, the molecular mechanisms orchestrating diurnal processes are still unknown in diatoms and many other phytoplanktonic organisms. Notably, no orthologs of the circadian clock components discovered in bacteria, fungi, animals or plants have been found in the diatom genomes except for cryptochromes (33). Nonetheless, a number of proteins containing bHLH-PAS domains, which feature in genes involved in the regulation of rhythmic processes in animals (34), have been identified in diatom genomes (35).

In this work, we integrate transcriptomic, physiological and functional analyses to explore the regulatory landscape of $P$. tricornutum diurnal rhythms. We uncover the function of a bHLH-PAS protein (PtbHLH1a) in the regulation of critical light-dependent rhythmic processes, such as cell cycle and diurnal transcription. Phylogenetic analyses reveal that 
bHLH1a homologs are widely distributed in diatoms, thus we speculate a common function in many diatom species. These results open the way to new exploration of diatom genomes in search of their elusive molecular timekeepers.

\section{RESULTS}

\section{Transcriptome profiling identifies potential regulators of diurnal rhythms in $P$.} tricornutum

To identify potential regulators of cellular rhythmicity in $P$. tricornutum, a publicly available diurnal transcriptomic dataset (27) was analyzed. One hundred and four genes with robust diel oscillating expression were selected, of which eight were photoreceptors $(30,33,36,37)$ and 66 were transcription factors (TFs) (35), which might be involved in the generation of lightdependent rhythms. The remaining 30 genes selected were potential output genes implicated in diel rhythmic processes (pigment synthesis, cell cycle regulation and photosynthesis) (Table S1). The transcriptional dynamics of the selected genes were examined in a 16:8-h light:dark (L:D) photocycle for 32h. Hierarchical clustering (HCL) analysis of the resulting expression profiles revealed 4 clusters of co-expressed genes, termed A-D (Fig. 1A), with peak expression at different times between dawn and dusk (Fig. 1B). Cluster A phased at dawn, suggesting a transcriptional anticipation of the light onset (Fig. 1A-B). This cluster comprised 18 genes including 14 TFs, mostly belonging to the Heat Shock Transcription Factor family (HSF), two DNA repair enzymes CPD photolyases (CPD2 and CPD3) and one carotenoid synthesis enzyme (PDS1). Cluster B phased around 7h Zeitgeber Time (hours after illumination, ZT) and encompassed 36 genes (Fig. 1A-B), including the dsCYC2 gene controlling the onset of cell division (30). Cluster B also contained 18 TFs, of which eight were sigma factors putatively involved in the regulation of chloroplast transcription, three genes implicated in photoprotection (LHCX1, Vdl2 and Zepl) and the chlorophyll synthesis PORl gene. Such active transcription of genes involved in chloroplast activity during the light period has been shown previously $(21,38)$. The blue light sensors Aurochromelb and the cryptochromes $C P F 1$ and $C r y P$-like also belonged to cluster B and show a strong expression following light onset, in accordance with previous observations (39-41). Cluster C phased around ZT9 (Fig. 1A-B) and comprises 9 TFs and 10 metabolism-related genes, including genes encoding photosynthetic apparatus. Finally, cluster D phased before dusk and included $23 \mathrm{TF}$ genes including the TCA cycle regulator $\operatorname{bZIP14}(27,32)$, likely contributing to preparing cells for light to dark transition (Fig. 1A-B). Cluster D also contained the CPF4 and 
132 the Far-Red light sensing phytochrome (DPH1) whose peak expression at the end of the light 133 period has been observed previously $(36,39)$.

134 Together these results underline the existence of tight transcriptional programs phasing at discrete moments of the day which potentially control the timing of cellular activities along the diurnal cycle.

\section{PtbHLHIa expression is adjusted in a photoperiod-dependent manner}

Our analysis identified two TFs, PtbHLH1a (Phatr3_J44962) belonging to cluster D and PtbHLH1b (Phatr3_J44963) belonging to cluster C, which each have a Per-ARNT-Sim (PAS) domain in conjunction with a bHLH DNA-binding domain. Because bHLH-PAS proteins have been shown to be involved in the regulation of rhythmic processes in animals $(4,34,42)$, the expression profiles of PtbHLHIa and PtbHLHIb were examined in P. tricornutum cells growing under different photoperiods. PtbHLHla expression peaked at ZT8 in the 12L:12D photoperiod and at ZT12 in the 16L:8D photoperiod, 4 hours before the end of the light period in both cases, then gradually decreased to below detection limits at ZT0 (Fig. 1C). Transcription of PtbHLHIb appeared to start earlier than that of PtbHLHIa. In cells entrained in 12L:12D cycles, PtbHLH1b expression peaked at ZT8, whereas it peaked between ZT8/ZT12 in 16L:8D photoperiods (Fig. 1C). Thus, PtbHLH1b expression onset almost coincided in the two photoperiods although transcription dramatically dropped after ZT8 in 12L:12D, while remained at maximum levels up to ZT12 in long days (Fig. 1C).

The robustness of PtbHLHIa and PtbHLH1b diurnal expression profiles was further examined under stress conditions using recent transcriptome datasets from $P$. tricornutum cells grown in 12L:12D cycles in iron replete and deplete conditions (28). Iron homeostasis is diurnally regulated in phytoplankton (43) and it affects rhythmic processes such as cell cycle progression and diurnal gene expression in P. tricornutum (28). Interestingly, PtbHLHIa and PtbHLH1b expression profiles showed similar patterns in both control and iron depleted conditions, with peaks of expression before dusk at ZT9 (Fig. S1), similar to our observations (Fig. 1C).

Altogether these results demonstrate robust control of PtbHLHIa and PtbHLHIb diurnal expression timing, which is adjusted in a photoperiod-dependent manner and unaffected by iron depletion. The involvement of PtbHLH1a in the regulation of diurnal light-dependent rhythmic processes was hypothesized considering a possible role in dusk anticipation. 
PtbHLHIa ectopic expression determines phase shifts in cellular rhythmicity

To determine PtbHLH1a's function, cell lines were generated expressing HA-tagged bHLH1a under the regulation of the Light harvesting complex protein family F2 promoter (Lhcf2p) (Fig. 2A), which activates transcription 3h after light onset (44). Gene expression analysis allowed the selection of three independent lines, hereafter named OE-1, OE-2 and OE-3, showing over-expression of the PtbHLHIa gene (Fig. 2B) and earlier expression timing compared to the wild type (Wt) strain (Fig. 4A and S2). Next, daily cellular rhythmicity was analyzed using the flow cytometer channel FL3 (excitation 488nm, emission $655-730 \mathrm{~nm})$ that estimates chlorophyll a cellular content $(8,38)$, over the course of three days (Fig 2C). Cellular fluorescence displayed highly oscillating rhythms in 16L:8D grown cultures with a periodicity of approximately $24 \mathrm{~h}$ (Fig. 2C, Table S2). Cell fluorescence in Wt cultures increased during daytime to peak around ZT13 (Fig. 2D and Table S2), and then started to decrease before night onset. This fall in fluorescence was concomitant with an increase in cell concentration ((38) and Fig. S4), likely reflecting chloroplast partitioning to daughter cells during cell division (7). Fluorescence progressively declined during the night period, reaching a trough in the early morning (Fig. 2C). Despite maintaining rhythmicity in the cellular fluorescence dynamics, OE-1 (Fig 2C), as well as OE-2 and OE-3 lines (Fig. 2D), displayed a remarkable phase shift of around 1-2 $\mathrm{h}$ in the maximum fluorescence timing compared to the $\mathrm{Wt}$ (Table S2). Cellular fluorescence phase responses were further investigated in resetting experiments. Wt and OE-1 cultures, that show the strongest phase shift phenotype, were grown in 16L:8D photoperiods, then transferred to $8 \mathrm{~L}: 16 \mathrm{D}$ and monitored for another 6 days. After the transfer to 8L:16D photocycles, the timing of maximum cell fluorescence in Wt cells was maintained for two days and then re-synchronized to the newly imposed photoperiod, peaking at ZT7.99 \pm 1.88 starting from the third day (Fig. 2E-F). In contrast, after 3 days of re-entrainment in $8 \mathrm{~L}: 16 \mathrm{D}$, the OE-1 line showed a 3-hour phase delay (ZT 11.49 \pm 2.87 ) compared to Wt (Fig. 2E-F). Together, these results indicate that diatom cellular rhythmicity is entrained in a photoperiod-dependent manner and that $P t \mathrm{bHLH1}$ a deregulation alters the capacity of cells to set diurnal phase pattern.

\section{PtbHLH1a regulates diurnal cell cycle progression}

The altered rhythm of fluorescence upon PtbHLHIa over-expression described above may reflect delayed or asynchronous cell division dynamics. To get further insights into the effect of PtbHLHIa overexpression on rhythmic processes, cell cycle dynamics in the WT and OE-1 lines were thoroughly analysed. Cell cultures were synchronized by $40 \mathrm{~h}$ of dark treatment and 
harvested on an hourly basis for $12 \mathrm{~h}$ after re-illumination. At T0, total DNA content measurements showed comparable proportions of cells in G1 phase in all samples indicating effective synchronization of cell cultures (Fig. 3A). Starting after $3 \mathrm{~h}$ of illumination, a progressive reduction of $\mathrm{G} 1$ cell number was observed in the $\mathrm{Wt}$, with a minimum number reached after 10h of illumination. After 10h, the percentage of Wt cells in G1 increased with the emergence of daughter cells (Fig. 3A). Interestingly, compared to Wt, OE-1 cells showed a slower exit from the G1 phase, with the percentage G1 cells continuing to decrease over the entire $12 \mathrm{~h}$ of illumination studied (Fig. 3A).

To further characterize the cell cycle deregulation caused by PtbHLHIa over-expression, the expression profiles of specific cell cycle phase marker genes (31) were analyzed in darksynchronized Wt and OE-1 lines illuminated for $12 \mathrm{~h}$. The G1 phase gene markers CDKA1 and $C D K D 1$ showed similar expression profiles in both lines until $4 \mathrm{~h}$ from the onset of illumination (Fig. 3B). Starting from this time point, transcript levels of both genes were consistently higher in the OE-1 line compared to Wt except for at the end of the time course when they converged. This illustrates that the G1 phase duration of the two cell lines is different. Conversely, the G2/M marker CYCB1 showed lower expression in OE-1 compared to the Wt between 4 and $8 \mathrm{~h}$ after the onset of illumination (Fig. 3B). The expression of another $\mathrm{G} 2 / \mathrm{M}$ phase marker, $C Y C A / B 1$, also resulted deregulated in $\mathrm{OE}-1$, presenting reduced amplitude and peaking $2 \mathrm{~h}$ later compared to $\mathrm{Wt}$. Together, these results suggest that the deregulation of PtbHLHIa affects cell cycle, possibly by altering transition from G1 to $\mathrm{S}$ or $\mathrm{G} 2 / \mathrm{M}$ phases.

\section{PtbHLH1a regulates pace of diel gene expression}

The effect of PtbHLH1a de-regulation on gene expression was investigated since the expression of many P. tricornutum genes phase diurnally (27). To this end, Wt and OE-1 lines were grown in 16L:8D photocycles and sampled every 3 hours over 25 hours. For this analysis, genes with strong diurnal transcription oscillation were selected, including TFs (bHLH1a, bHLH1b and bHLH3) and rhythmic genes putatively involved in chlorophyll and carotenoid synthesis (NADPH:protochlorophyllide oxidoreductase 2, Por2, and Violaxanthin de-epoxidase-related, Vdr) (Fig. 1A, $(27,38)$ ). Total PtbHLHla transcript levels, including endogenous and transgenic mRNAs, were shown to be higher in OE-1 cells compared to the $\mathrm{Wt}$, and the expression peaking at ZT7 in the OE-1 line and ZT10 in the Wt (Fig. 4A). A

231 decrease of endogenous PtbHLHla transcripts was observed in the OE-1 line compared to the 232 Wt, possibly reflecting negative feed-back mechanism of PtbHLH1a regulating its own 
transcription (Fig. 4A). A similar pattern was also observed for the PtbHLH1b gene, suggesting that PtbHLHIa and PtbHLHIb transcription is controlled by the same regulatory circuit. In addition, the $b H L H 3$ gene showed earlier phases of expression in OE-1 compared to the Wt (Fig. 4A). Similar deregulations of PtbHLHIa, PtbHLH1b PtbHLH3 were also observed in the OE-2 and OE-3 lines at ZT10 (Fig. S3). Besides TFs, the Chlorophyll biosynthesis gene Por2 was also anticipated and the $V d r$ gene presented increased amplitude of expression in the OE-1 line compared to the Wt (Fig. 4A).

Altered gene expression observed in PtbHLH1a overexpression cell lines could be the consequence of the deregulation of cell cycle progression (Fig. 3). To test this hypothesis, gene expression was analyzed in dark conditions, when the cell cycle is arrested ((31) and (Fig. S5)). Because information about transcription dynamics in this condition was limited, an initial survey of expression of the previously selected 104 P. tricornutum diurnal rhythmic genes (Fig. 1A) was performed in cells exposed to continuous dark for 30 hours. Comparative analysis of transcript profiles revealed that around $20 \%$ of the genes show persistent oscillation of expression in D:D, although in some cases they displayed reduced amplitudes and/or shifted phases of expression compared to the 16L:8D condition. In particular, 19 genes were identified which showed the highest amplitude of expression in both L:D and D:D (for details see Materials and Methods), consisting of 16 putative TFs and 4 pigment-related enzymes (Fig. S6). Among the analyzed transcripts, genes that were severely affected by the absence of light were also found, being strongly down-regulated or over-expressed when compared to the L:D condition (Fig. S7). The expression of some of these genes was further analyzed in constant darkness in Wt and PtbHLH1a OE-1 cells for a period of $24 \mathrm{~h}$. In the Wt, the analyzed genes showed comparable transcript profiles in D:D and 16L:8D conditions (Fig. 4B, Fig. S8). Conversely, 10 out of 13 tested genes displayed reduced amplitudes and shifts in the phase of expression in OE-1 compared to Wt in D:D (Fig. 4B, Fig. S8). It is worth mentioning that two of the analysed genes, HSFId and bZIP5, showed almost overlapping profiles in OE-1 and Wt lines (Fig. S8), excluding global deregulation of transcription by modulation of bHLH1a expression. Taken together, these results suggest that PtbHLH1a contributes to define timing of diurnal gene expression and that its activity is independent of direct light inputs and cell division.

\section{PtbHLH1a-like proteins are widely represented in the genome of marine algae} until genome and transcriptome sequencing projects revealed bHLH-PAS family members in 
diatoms (35) and other microalgae (46). Interestingly, when compared to animal bHLH-PAS, diatom proteins show peculiar features including a single predicted PAS domain (Fig. 5A), whereas animal bHLH-PAS proteins have two, and a N-ter extension that is absent in the animal counterparts. Available transcriptomic and genomic databases of marine algae and animals were searched for bHLH-PAS proteins and $\approx 90$ novel bHLH-PAS proteins were discovered from Rhodophyta, Cryptophyta, Stramenopila, Alveolata and basal Opistokonta organisms (Table S3). With one exception, all the newly identified proteins showed a single predicted PAS domain, short $\mathrm{C}$-ter extensions and $\mathrm{N}$-ter regions of variable length, similar to the predicted structure of diatom bHLH-PAS proteins (Fig. 5A). Notably, the only bHLHPAS possessing two PAS domains like the animal proteins was identified in Galdieria sulphuraria (Rhodophyta) and represents the first TF of this family identified in Archaeplastida. All the identified sequences, including selected bHLH-PAS from Opistokonta lineages, were used to perform a detailed phylogenetic analysis of the protein family using the bHLH and PAS domains. This analysis revealed at least three clades of algal bHLH-PAS proteins clearly separated from their Opistokonta counterparts (Fig. 5B). Interestingly, domain organization and branching positions of proteins from basal Opistokonta (Monosiga brevicollis) and microalgae (Guillardia theta (Cryptophyta) and Nannochloropsis (Stramenopila)) (Fig. 4B) support a possible common origin for this TF family, from an ancestor featuring single bHLH and PAS domains. However, the possible contribution of horizontal gene transfer and convergent evolution to the proliferation and diversification of this family cannot be excluded, and may be supported by the features of the G. sulphuraria bHLH-PAS protein, likely independently acquired by this alga. Based on our analysis, the majority of microalgal bHLH-PAS proteins fall into three separate clades: the first containing 9 TFs from diatoms and Ectocarpus siliculosus, the second comprising PtbHLH1a together with 35 proteins from diatoms and Alveolata (Dinoflagellata), and the third comprising 41 proteins from Alveolata (Ciliophora and Dinoflagellata) and diatoms, including PtbHLH1b (Fig. 5B).

Our results highlight diversification and widespread distribution of bHLH-PAS family members in different groups of algae. Moreover, the presence of bHLH1a-like genes in the genome of dinoflagellate and diatom species suggests that these proteins may share similar functions in microalgae. The similarities in diel transcript regulation and timing of expression between PtbHLH1a and the centric diatom Thalassiosira pseudonana ortholog, TpHLH1 (26), 


\section{DISCUSSION}

The diurnal cycle is characterised by profound periodic light and temperature changes which have shaped the evolution of most ecosystems on Earth. In most organisms, biological rhythms are controlled by interconnected transcriptional-translational feed-back loops involving TFs and integrating signals from the environment (5). Although this regulatory framework is conserved among eukaryotes, the regulators responsible for the timing of events within biological rhythms seem to have emerged several times through evolution (47). Therefore, our current understanding of diurnal and circadian regulation, largely based on the study of terrestrial model organisms, is not always appropriate or relevant for evolutionarily distant marine organisms. In this study, we have shed light on the unknown regulators of diurnal patterns in diatoms, one of the most prominent phytoplanktonic groups in the Ocean. In agreement with previous studies (27, 28), we showed the existence of organized transcriptional programs defining cellular activities along the daily cycle in $P$. tricornutum and identified a number of TFs phasing at different times during the $24 \mathrm{~h}$ cycle, as novel candidates for diatom diurnal regulation. By monitoring diurnal variations in chlorophyll fluorescence, robust regulation of diatom physiological rhythms that can be re-entrained to changing photoperiods was also unveiled. The ability to adjust the phase according to the photoperiod length constitutes one defining criterion of circadian clock-regulated mechanisms (48). Likewise, the strongly oscillating diel expression pattern of a P. tricornutum bHLH-PAS gene, PtbHLHla, responded to photoperiod length with peak expression 4 hours before night onset in both $12 \mathrm{~h}$ and $16 \mathrm{~h}$ day photoperiods. The timing of PtbHLHIa expression is also preserved in cells under iron deficiency, in contrast to the expression of many other $P$. tricornutum genes observed previously, and despite the growth rate reductions caused by nutriment depletion (28). Functional characterization of PtbHLH1a established its involvement in the regulation of $P$. tricornutum diurnal rhythms. Transgenic lines overexpressing PtbHLHIa using a promotor that is activated earlier in the light period than the endogenous gene maintained cellular rhythms of $\sim 24 \mathrm{~h}$ but show phase-alterations that are even more accentuated in re-entrainment experiments. This phenotype may reflect a reduced capacity of cells to synchronize to environmental light-dark cycles and adjust the phase to the new photoperiod. The participation of bHLH1a in the regulation of $P$. tricornutum cell cycle progression, reported in this study, could also explain the altered cellular fluorescence rhythmicity observed in the mutants. Altered cell division timing could derive from a delayed exit from the G1 phase in transgenic lines compared to $\mathrm{Wt}$, as also supported by the altered 
expression of the mitotic cyclins CYCB1 and CYCA/B1 in these lines (31). The PtbHLH1a gene could participate in gating cell divisions at night time, therefore maximizing the energetic budget, as observed in several unicellular algae $(7,8,30,49)$. Interestingly, a similar regulation of the cell cycle occurs in mammalian cells, where the circadian clock controls the expression of G2 cycle-related genes to gate cell division at specific times of the day (50).

Besides cell cycle, PtbHLH1a deregulation also affected diurnal rhythmicity of several gene transcripts. This phenotype was uncoupled from cell cycle deregulation as it was observed also in the absence of cell division, during darkness. Interestingly, the deregulation of gene transcription was much more pronounced when analyzed in D:D compared to L:D conditions. These results suggest on one hand that multiple regulatory inputs participate in the regulation of diurnal rhythmic gene transcription (48), partially masking PtbHLH1a contribution to this process in cyclic environments, and, on the other hand, support PtbHLH1a involvement in the maintenance of rhythms in the absence of light inputs.

The evidence provided in this work support the hypothesis that PtbHLH1a is one component of an uncharacterized endogenous circadian clock in diatoms, either as part of a central oscillator or as a mediator of clock inputs or outputs. With the exception of CRY (33, 39), orthologs of plant and animal circadian clock genes are absent in diatom genomes. However, PtbHLH1a contains bHLH and PAS protein domains that are also present in the CLOCK and BMAL proteins, components of the mammalian central circadian oscillator (51, 52). Interestingly, previous studies showed that the $P$. tricornutum animal-like blue light sensor Cpf1 can repress the transcriptional activity of these proteins in a heterologous mammalian cell system (33), suggesting at least partial conservation in the regulatory program generating rhythmicity in animals and diatoms. The downregulation of endogenous PtbHLHIa and PtbHLH1b transcripts in the OE lines analysed in this work likely reflects a negative feedback loop in the regulation of these genes, which is typical of circadian genetic oscillators, and further supports a possible role for PtbHLHIa in diatom rhythm regulation. Moreover, as observed for the clock components, we show that PtbHLH1a contributes to set the phase of output processes in cycling environments and to the maintenance of gene expression rhythmicity in constant darkness. Some TFs characterized in this study and showing altered expression patterns in PtbHLH1a transgenic cells (i.e., bHLH1b, bHLH3, bZIP5, bZIP7, HSF1d, HSF1g, HSF3.3a, HSF4.7b) represent direct or indirect targets of PtbHLH1a activity and possible additional components of the network participating in diel rhythm regulation. PtbHLH1a might act downstream of signal transduction cascades activated by the diatom photoreceptors analysed in this study and elsewhere. The presence of a PAS 
domain in PtbHLH1a also suggests that this protein might have its own light-sensing ability (53).

Although further analyses under prolonged free running conditions (for example continuous darkness and continuous illumination) will be necessary to conclusively assess the involvement of PtbHLH1a in circadian regulation, this protein constitutes a promising entry point for the characterization of diatom molecular timekeepers. Finally, the discovery of the wide distribution of bHLH-PAS domain-containing proteins in diatoms, as well as in other algae, has the potential to shed new light on the evolution of biological rhythms. bHLH-PAS proteins might have independently acquired their function in rhythm regulation by convergent evolution. However, the existence of this function in an ancient heterotrophic marine ancestor that subsequently acquired plastids via endosymbiosis events (54) and prior to colonization of land cannot be excluded. Regulators of cellular rhythmicity such as PtbHLH1a may have played a critical role for diatom prominence in marine ecosystems, by synchronizing cellular activities in optimal temporal programs and maximizing diatoms' ability to anticipate and adapt to cyclic environmental variations.

Biological rhythms are still poorly understood at molecular and mechanistic levels in marine algae, despite their fundamental significance to these organisms' biology and ecology.

386 Further characterization of PtbHLH1a homologs in diatoms and other algae is expected to 387 provide new insights into biological rhythms in marine organisms.

\section{METHODS}

\section{Culture conditions}

391 Wild-type P. tricornutum (Pt1 8.6; CCMP2561) cells and transgenic lines were grown at $39218^{\circ} \mathrm{C}$ with shaking at $100 \mathrm{rpm}$ in $\mathrm{F} / 2$ medium (55) without silica and illuminated at $40 \mu \mathrm{mol}$ 393 photons $\mathrm{m}^{-2} \mathrm{~s}^{-1}$ of white light (Philips TL-D De Luxe Pro 950). Detailed information is in SI.

\section{Cell cycle analysis}

396 Cells were synchronized in the G1 phase by 40h of darkness. After re-illumination, samples 397 were collected every hour for $12 \mathrm{~h}$. Details are in SI. 
RNA extraction and gene expression analyses

400 Total RNA was extracted and qRT-PCR performed as described in (36). Codeset information 401 and raw nCounter data are available from the GEO database (Series GSE112268). Detailed 402 information is in SI.

403

\section{Selection of rhythmic transcripts and clustering analysis}

405 To select genes with rhythmic expression in the light-dark cycle, we used microarray data 406 from (27). To select rhythmic transcripts in D:D, standard deviation from the average 407 expression were calculated and used as selective criteria. Detailed information is in SI.

408

409 Generation of the PtbHLH1a overexpressing lines

410 Diatom transgenic lines were obtained by co-transformation of the pDEST-C-HA-PtbHLHIa

411 plasmid together with Nourseothricin resistance plasmid. Details are described in SI.

413 Data mining, protein sequence and phylogenetic analysis

414 Detailed information about data mining, protein sequence and phylogenetic analysis is 415 provided in SI.

416

\section{ACKNOWLEDGEMENTS}

418 We thank M. Jaubert and L. De Veylder for critical suggestions, A. Manzotti for help with 419 characterization of OE lines, P. Oliveri for support with nCounter technology, D. Petroutsos 420 and G. Finazzi for support in monitoring cell physiology. This work was funded by the HFSP 421 research grant (\#RGY0082/2010), EMBRIC, EMBRC-FR and a grant from the Gordon and 422 Betty Moore Foundation (GBMF 4966) to A.F. 


\section{REFERENCES}

1. Pittendrigh CS (1993) Temporal Organization: Reflections of a Darwinian Clock-Watcher. Annual Review of Physiology 55(1):17-54.

2. Dodd AN, et al. (2005) Plant circadian clocks increase photosynthesis, growth, survival, and competitive advantage. Science 309(5734):630-633.

3. Woelfle MA, Ouyang Y, Phanvijhitsiri K, \& Johnson CH (2004) The adaptive value of circadian clocks: an experimental assessment in cyanobacteria. Curr Biol 14(16):1481-1486.

4. Dunlap JC (1999) Molecular bases for circadian clocks. Cell 96(2):271-290.

5. Bell-Pedersen D, et al. (2005) Circadian rhythms from multiple oscillators: lessons from diverse organisms. Nat Rev Genet 6(7):544-556.

6. Chisholm SW (1981) Temporal Patterns of Cell-Division in Unicellular Algae. Can B Fish Aquat Sci (210):150-181.

7. Moulager M, et al. (2007) Light-dependent regulation of cell division in Ostreococcus: evidence for a major transcriptional input. Plant physiology 144(3):1360-1369.

8. Ragni M \& D'Alcala MR (2007) Circadian variability in the photobiology of Phaeodactylum tricornutum: pigment content. J Plankton Res 29(2):141-156.

9. Naylor E (2010) Chronobiology of marine organisms (Cambridge University Press, Cambridge; New York) pp x, 242 p.

10. Tessmar-Raible K, Raible F, \& Arboleda E (2011) Another place, another timer: Marine species and the rhythms of life. Bioessays 33(3):165-172.

11. Ottesen EA, et al. (2013) Pattern and synchrony of gene expression among sympatric marine microbial populations. P Natl Acad Sci USA 110(6):E488-E497.

12. Brierley AS (2014) Diel vertical migration. Current Biology 24(22):R1074-R1076.

13. Hafker NS, et al. (2017) Circadian Clock Involvement in Zooplankton Diel Vertical Migration. Current Biology 27(14):2194-2201.

14. Field CB, Behrenfeld MJ, Randerson JT, \& Falkowski P (1998) Primary production of the biosphere: integrating terrestrial and oceanic components. Science 281(5374):237-240.

15. Malviya $S$, et al. (2016) Insights into global diatom distribution and diversity in the world's ocean. Proc Natl Acad Sci U S A 113(11):E1516-1525.

16. Baldauf SL (2003) The Deep Roots of Eukaryotes. Science 300(5626):1703-1706.

17. Bowler C, et al. (2008) The Phaeodactylum genome reveals the evolutionary history of diatom genomes. Nature 456(7219):239-244.

18. Moustafa A, et al. (2009) Genomic footprints of a cryptic plastid endosymbiosis in diatoms. Science 324(5935):1724-1726.

19. Flori S, et al. (2017) Plastid thylakoid architecture optimizes photosynthesis in diatoms. Nat Commun 8:15885.

20. Raible F \& Falciatore A (2014) It's about time: Rhythms as a new dimension of molecular marine research. Mar Genomics 14:1-2.

21. Bailleul B, et al. (2010) An atypical member of the light-harvesting complex stress-related protein family modulates diatom responses to light. P Natl Acad Sci USA 107(42):1821418219.

22. Allen AE, et al. (2011) Evolution and metabolic significance of the urea cycle in photosynthetic diatoms. Nature 473:203.

23. Goss R \& Lepetit B (2015) Biodiversity of NPQ. Journal of Plant Physiology 172:13-32.

24. Morrissey J, et al. (2015) A novel protein, ubiquitous in marine phytoplankton, concentrates iron at the cell surface and facilitates uptake. Curr Biol 25(3):364-371.

25. McQuaid JB, et al. (2018) Carbonate-sensitive phytotransferrin controls high-affinity iron uptake in diatoms. Nature 555(7697):534-537.

26. Ashworth J, et al. (2013) Genome-wide diel growth state transitions in the diatom Thalassiosira pseudonana. P Natl Acad Sci USA 110(18):7518-7523.

27. Chauton MS, Winge P, Brembu T, Vadstein O, \& Bones AM (2013) Gene regulation of carbon fixation, storage, and utilization in the diatom Phaeodactylum tricornutum acclimated to light/dark cycles. Plant physiology 161(2):1034-1048. 
28. Smith SR, et al. (2016) Transcriptional Orchestration of the Global Cellular Response of a

29. Zones JM, Blaby IK, Merchant SS, \& Umen JG (2015) High-Resolution Profiling of a Synchronized Diurnal Transcriptome from Chlamydomonas reinhardtii Reveals Continuous Cell and Metabolic Differentiation. Plant Cell 27(10):2743-2769.

30. Huysman MJ, et al. (2013) AUREOCHROME1a-mediated induction of the diatom-specific cyclin dsCYC2 controls the onset of cell division in diatoms (Phaeodactylum tricornutum). Plant Cell 25(1):215-228.

31. Huysman MJ, et al. (2010) Genome-wide analysis of the diatom cell cycle unveils a novel type of cyclins involved in environmental signaling. Genome Biol 11(2):R17.

32. Matthijs M, et al. (2017) The transcription factor bZIP14 regulates the TCA cycle in the diatom Phaeodactylum tricornutum. The EMBO journal 36(11):1559-1576.

33. Coesel S, et al. (2009) Diatom PtCPF1 is a new cryptochrome/photolyase family member with DNA repair and transcription regulation activity. EMBO Rep 10(6):655-661.

34. Kewley RJ, Whitelaw ML, \& Chapman-Smith A (2004) The mammalian basic helix-loophelix/PAS family of transcriptional regulators. Int J Biochem Cell Biol 36(2):189-204.

35. Rayko E, Maumus F, Maheswari U, Jabbari K, \& Bowler C (2010) Transcription factor families inferred from genome sequences of photosynthetic stramenopiles. New Phytol 188(1):52-66.

36. Fortunato AE, et al. (2016) Diatom Phytochromes Reveal the Existence of Far-Red-LightBased Sensing in the Ocean. Plant Cell 28(3):616-628.

37. Jaubert M, Bouly JP, Ribera d'Alcala M, \& Falciatore A (2017) Light sensing and responses in marine microalgae. Curr Opin Plant Biol 37:70-77.

38. Hunsperger HM, Ford CJ, Miller JS, \& Cattolico RA (2016) Differential Regulation of Duplicate Light-Dependent Protochlorophyllide Oxidoreductases in the Diatom Phaeodactylum tricornutum. PLoS One 11(7):e0158614.

39. Fortunato AE, Annunziata R, Jaubert M, Bouly JP, \& Falciatore A (2015) Dealing with light: the widespread and multitasking cryptochrome/photolyase family in photosynthetic organisms. J Plant Physiol 172:42-54.

40. Oliveri P, et al. (2014) The Cryptochrome/Photolyase Family in aquatic organisms. Mar Genomics 14:23-37.

41. Banerjee A, et al. (2016) Allosteric communication between DNA-binding and lightresponsive domains of diatom class I aureochromes. Nucleic Acids Res 44(12):5957-5970.

42. Young MW \& Kay SA (2001) Time zones: a comparative genetics of circadian clocks. Nat Rev Genet 2(9):702-715.

43. Botebol H, et al. (2015) Central role for ferritin in the day/night regulation of iron homeostasis in marine phytoplankton. Proc Natl Acad Sci U S A 112(47):14652-14657.

44. Russo MT, Annunziata R, Sanges R, Ferrante MI, \& Falciatore A (2015) The upstream regulatory sequence of the light harvesting complex Lhcf2 gene of the marine diatom Phaeodactylum tricornutum enhances transcription in an orientation- and distance-independent fashion. Mar Genomics 24 Pt 1:69-79.

45. Yan J, Ma Z, Xu X, \& Guo AY (2014) Evolution, functional divergence and conserved exonintron structure of bHLH/PAS gene family. Mol Genet Genomics 289(1):25-36.

46. Thiriet-Rupert S, et al. (2016) Transcription factors in microalgae: genome-wide prediction and comparative analysis. BMC Genomics 17:282.

47. Bhadra U, Thakkar N, Das P, \& Bhadra MP (2017) Evolution of circadian rhythms: from bacteria to human. Sleep Med 35:49-61.

48. Merrow M, et al. (2006) Entrainment of the Neurospora circadian clock. Chronobiol Int 23(12):71-80.

49. Noordally ZB \& Millar AJ (2015) Clocks in algae. Biochemistry 54(2):171-183.

50. Matsuo T, et al. (2003) Control mechanism of the circadian clock for timing of cell division in vivo. Science 302(5643):255-259.

51. Bunger MK, et al. (2000) Mop3 is an essential component of the master circadian pacemaker in mammals. Cell 103(7):1009-1017. 
52. Gekakis N, et al. (1998) Role of the CLOCK protein in the mammalian circadian mechanism.

53. Taylor BL \& Zhulin IB (1999) PAS domains: internal sensors of oxygen, redox potential, and

light. Microbiol Mol Biol Rev 63(2):479-506.

54. Archibald JM (2015) Endosymbiosis and Eukaryotic Cell Evolution. Current biology 25(19):R911-921.

541

55. Guillard RRL ( 1975) Culture of Phytoplankton for Feeding Marine Invertebrates. In Culture 


\section{FIGURE LEGENDS}

547 Fig. 1. Diurnal expression analysis of selected rhythmic $\boldsymbol{P}$. tricornutum genes. A) 548 nCounter expression analysis of 104 selected genes in cells grown under a 16L:8D 549 photoperiod and sampled every 4 hours for 32 hours. Four major groups of co-regulated genes 550 (A-D) are shown based on hierarchical clustering. Expression values were normalized using 551 PtRPS, PtTBP and PtACTIN12 as reference genes and represent the average of three 552 biological replicates. The PtbHLHIa and PtbHLH1b gene expression profiles are indicated 553 with arrowheads. B) Polar plot and table showing the average phases of expression (intended 554 as expression peaks during the analyzed period) of the four gene clusters, calculated using the 555 MFourfit averaged method. Plot petal length is proportional to the standard deviation of 556 phases over biological replicates. In the table, N: number of genes within each cluster; Av. 557 Phase: average periods and phases of expression in hours. C) Diurnal expression profiles of PtbHLHIa and PtbHLH1b in Wt cells grown under 12L:12D (blue line) and 16L:8D (red line) photocycles analysed by qRT-PCR. Expression values were normalized using PtRPS and $P$ TTBP as reference genes and represent the average of three biological replicates \pm s.e.m (standard error of the mean; black bars).

562 For each gene, the expression value is relative to its maximum expression (maximum expression=1). Light and dark periods are represented by white and grey regions respectively.

Fig. 2. PtbHLH1a over-expression determines phase shifts in cellular rhythmicity.

A) Schematic representation of the Lhcf2p:bHLHla-3xHA:Lhcflt construct used to generate PtbHLH1a over-expressing lines. Lhcf2p: Light Harvesting Complex F2 promoter; 3HA: triple hemagglutinin tag; Lhcflt: Lhcfl terminator. B) Quantification of total PtbHLHla transcripts in OE-lines and Wt grown in 16L:8D photocycles and sampled at the ZT10. qRTPCR expression values were normalized using PtRPS and PtTBP as reference genes and represent the average of 3 biological replicates $(n=3) \pm$ s.e.m (black bars). C) Diurnal oscillation of chlorophyll fluorescence (FL-3 parameter) in Wt and OE lines entrained under 16L:8D over three days. D) Diurnal phase time calculation of the FL-3 value in Wt and OE-1,

574 OE-2 and OE-3 lines. Values represent the average of at least 4 biological replicates $(\mathrm{n} \leq 4)$ 575 from at least 2 independent experiments \pm s.e.m. E) Phase re-entrainment analysis of 576 fluorescence rhythms after photoperiod change from 16L:8D to 8L:16D in Wt (yellow) and 577 OE-1 (red). The FL-3 parameter was monitored in cultures grown in 16L:8D photocycles and 578 then transferred to $8 \mathrm{~L}: 16 \mathrm{D}$ for 6 days. Values represent the average of three biological 
replicates \pm s.e.m. F) Bar plot representation of the re-entrained FL-3 phases in Wt and OE-1

580 cultures after three 8L:16D photocycles. Values represent the average of 3 biological 581 replicates \pm s.e.m. (black bars).

$* \mathrm{P}<0.05, * * \mathrm{P}<0.01, * * * \mathrm{P}<0.001, \mathrm{t}$-test.

Fig. 3. PtbHLH1a over-expression affects cell cycle progression. A) Cell cycle progression dynamics of Wt (yellow) and OE-1 (red) lines shown as the proportion of cells in the G1 phase measured by flow cytometry each hour over $12 \mathrm{~h}$ of illumination following dark synchronization. Results are representative of three biological replicates \pm s.e.m (black bars); $\mathrm{t}$-test significance is indicated by *: $\mathrm{P}<0.05$, **: $\mathrm{P}<0.01$, t-test. B) qRT-PCR expression profiles of G1 (PtCDKA1, PtCDKD1) and G2/M phase marker genes (PtCYCB1, PtCYCA/B1) in synchronized Wt and OE-1 cell lines over $12 \mathrm{~h}$ of illumination. Expression values were normalized using PtRPS and PtTBP as reference genes and represent the average of two independent biological replicates. For each gene, the expression value is relative to its maximum expression (maximum expression=1).

Fig. 4 PtbHLH1a over-expression alters rhythmic diel gene expression. A) qRT-PCR diurnal expression analysis of PtbHLHIa, PtbHLH1b, PtbHLH3, PtPor2 and PtVdr transcripts in 16L:8D entrained Wt (yellow) and OE-1 (red) cultures. bHLH1a-total represents the sum expression of the bHLHIa endogenous and the transgene transcripts; PtbHLHla-endogenous refers to the endogenous gene only. Expression values represent the average of 2 biological replicates \pm s.e.m. (black bars). B) nCounter expression analysis of PtbHLH1a, PtbHLH1b, PtbHLH3, PtPor2 and PtVdr transcripts during 24 hours of dark freerunning period in the Wt (yellow) and OE-1 (red) lines. Cells were previously entrained in 16L:8D cycles. Data represent the average of 3 biological replicates \pm s.e.m (black bars).

604 Grey rectangles represent dark periods. Expression values were normalized using PtRPS and $P$ TTBP as reference genes. For each gene, the expression value is relative to its maximum expression (maximum expression $=1$ ).

Fig. 5. bHLH-PAS protein family structure and phylogeny. A) Schematic representation of bHLH-PAS protein domain architecture across Eukaryotes. Segmented line indicates possible absence of the second PAS domain in some Opistokonta species. The grey patterns before the bHLH domain and after the PAS domain represent the variations in N-ter and C-ter length in different organisms. B) Maximum Likelihood (ML) phylogenetic tree of the bHLH- 
613 PAS family. The Opistokonta clade is used as the outgroup. Numbers refer to bootstrap values 614 of the basal nodes using ML (RAxML, 1000 bootstraps) and Bayesian Inference (MrBayes, $6152.5 \mathrm{M}$ generations, $25 \%$ burn-in). The asterisk, the arrows and the square indicate the position 616 of Monosiga brevicollis bHLH-PAS, P. tricornutum HLH1a and bHLH1b, and Thalassiosira 617 pseudonana bHLH1, respectively. The colour code indicates the lineage corresponding to 618 each bHLH-PAS protein, shown in Fig 5A. 
bioRxiv preprint doi: https://doi.org/10.1101/271445; this version posted May 26, 2018. The copyright holder for this preprint (which was not certified by peer review) is the author/funder. All rights reserved. No reuse allowed without permission.

Figure 1 - Annunziata et al.

A
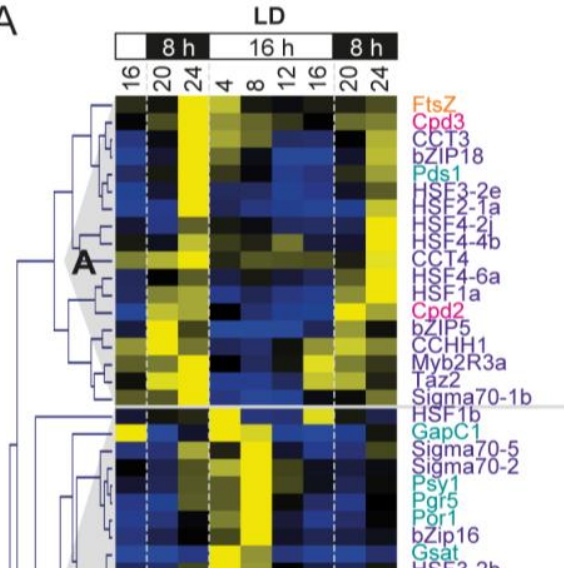

B

621

622

Photoreceptors TFs Cell Cyle

Metabolism

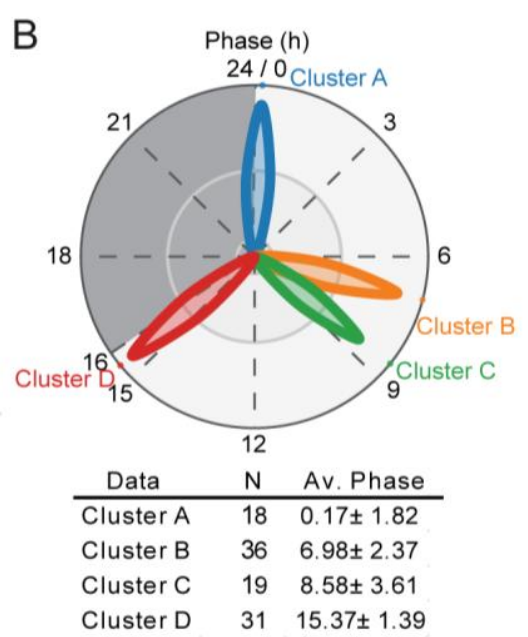

C
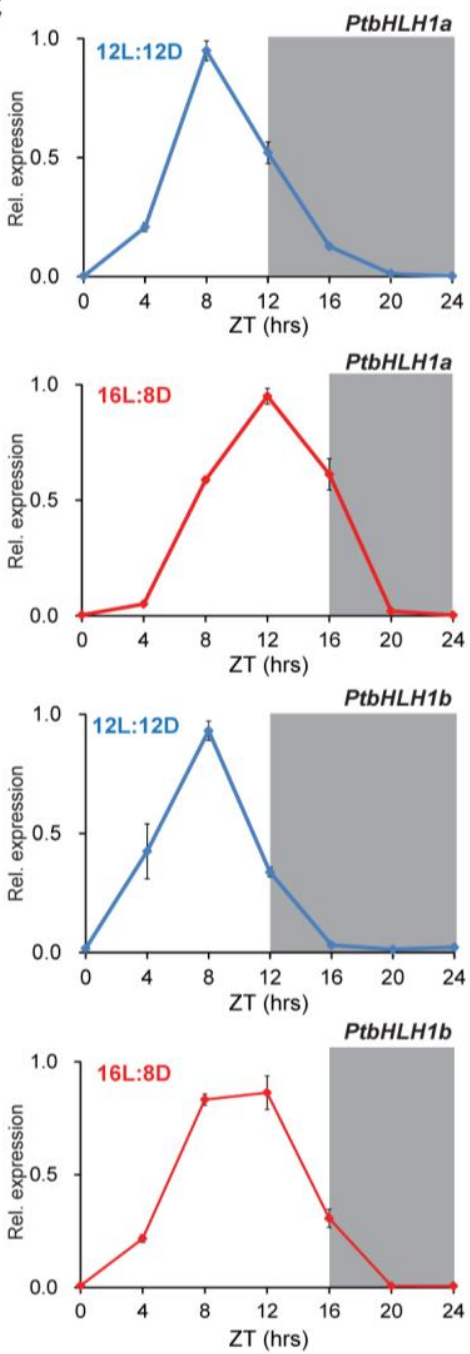
Figure 2 - Annunziata et al.
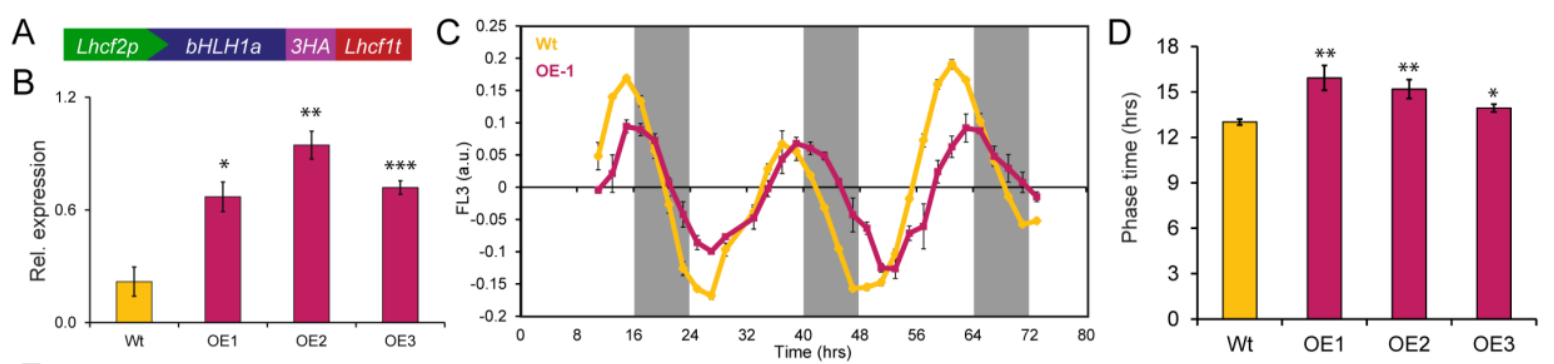

E

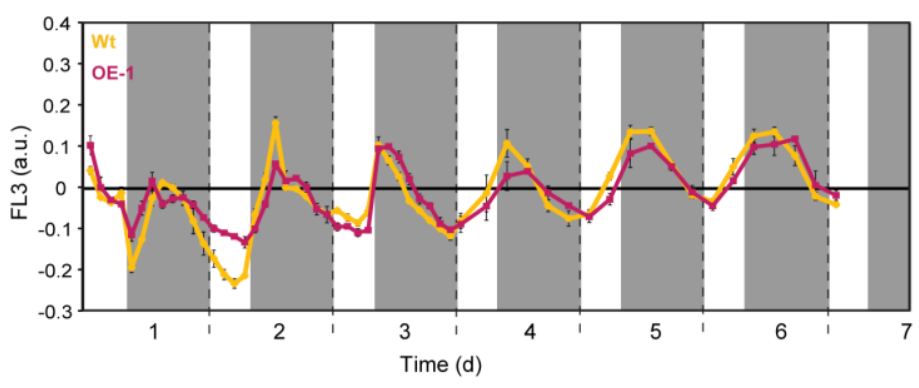

F

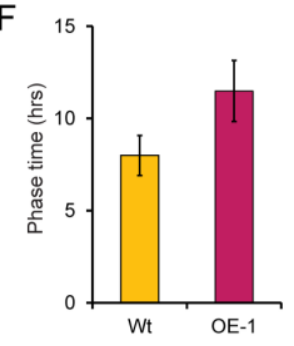




\section{Figure 3 - Annunziata et al.}
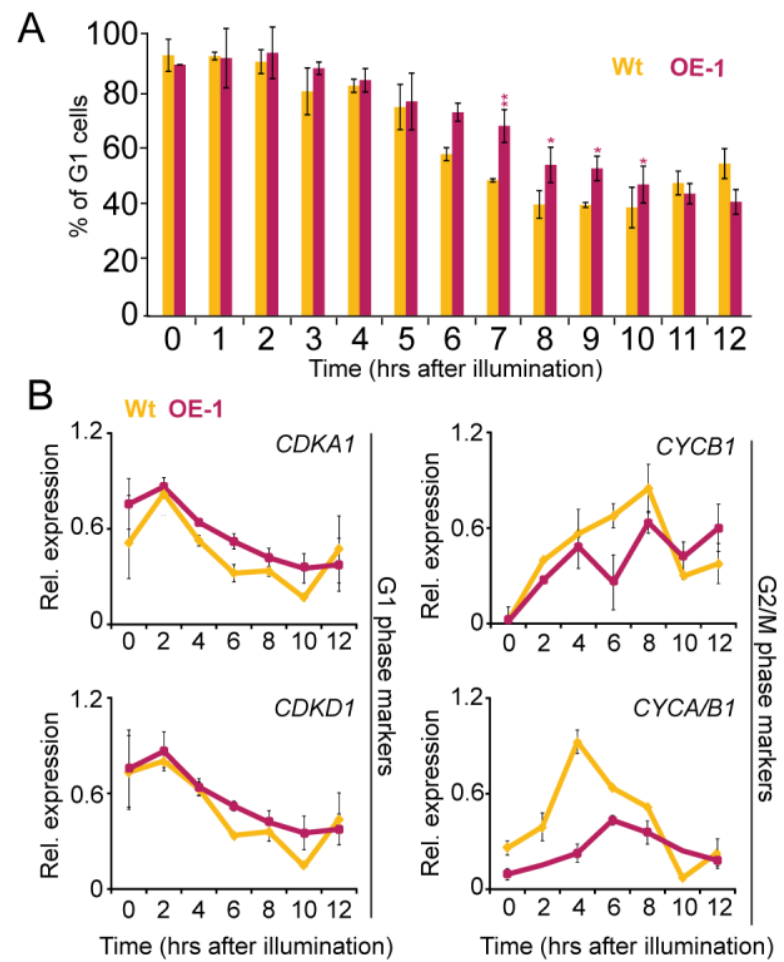
Figure 4 - Annunziata et al.

A
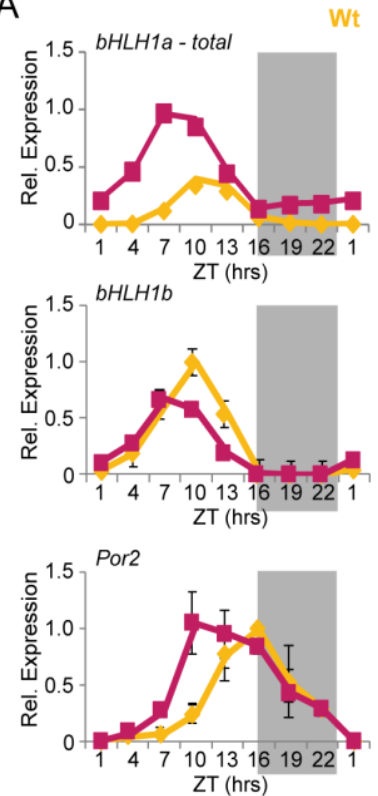

Wt $\mathrm{OE}-1$
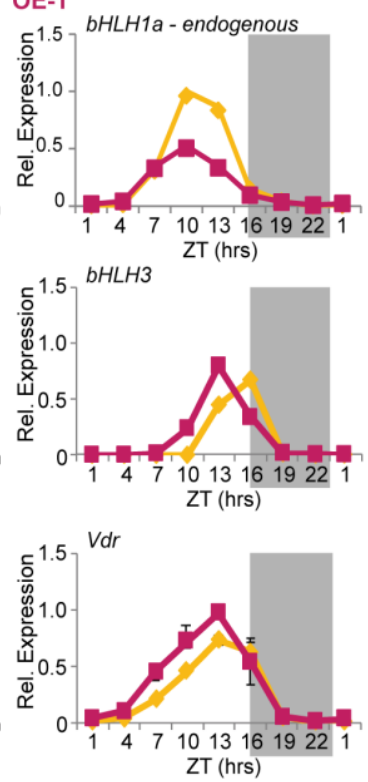

B
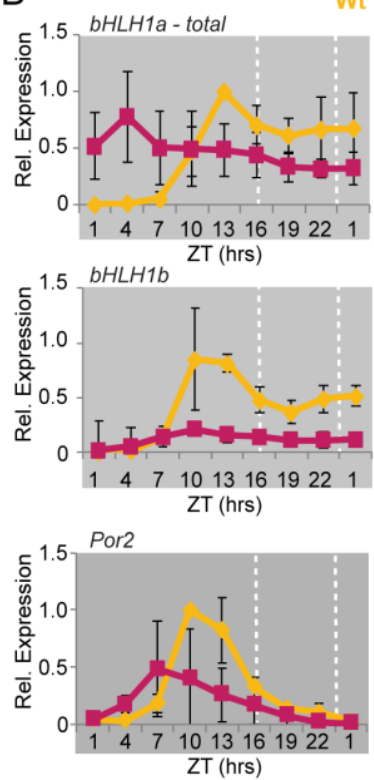

Wt OE-1
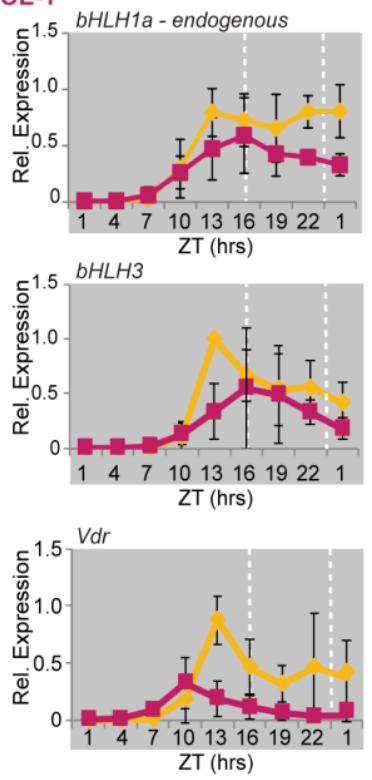

631

632 
Figure 5 - Annunziata et al.

A

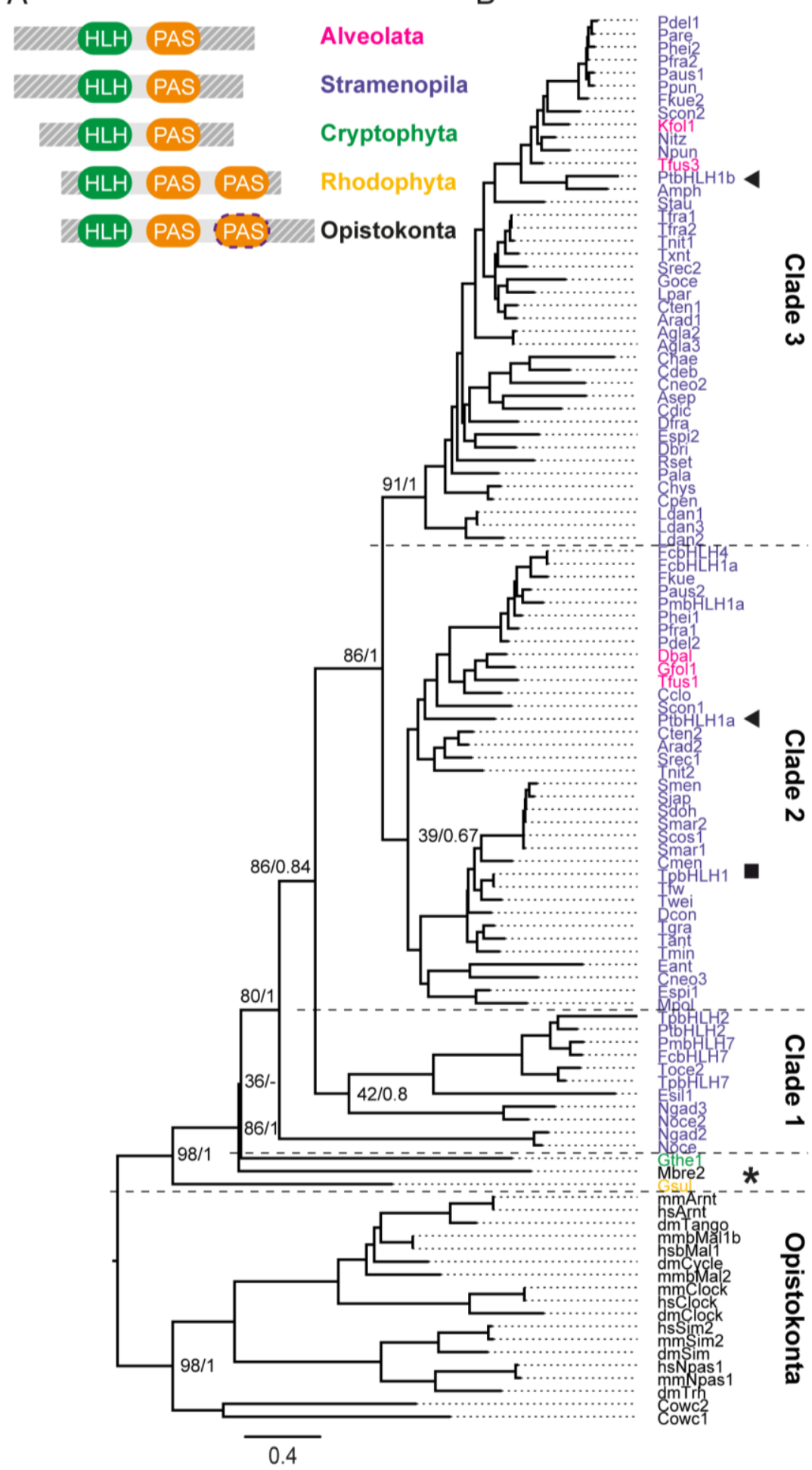




\section{Culture conditions}

641 For experiments in different photoperiods, cultures were pre-adapted to the different L:D 642 cycles for 2 weeks before starting the experiment. For experiments in continuous darkness 643 cells were pre-adapted in 16L:8D photocycles for 2 weeks, then transferred to D:D at the start 644 of the experiment. Growth measurements were performed using a MACSQuant Analyser flow 645 cytometer (Miltenyi Biotec, Germany) by counting the cells based on the R1-A (630nm 646 excitation, 670-700nm emission) versus the R1-H parameters. Phase rhythmicity assays were 647 carried out by measuring the Chlorophyll fluorescence using the flow cytometer FL-3 648 parameter (488 nm excitation, 670-700 nm emission). For the re-entrainment experiment, 649 cultures were initially entrained under $16 \mathrm{~L}: 8 \mathrm{D}$ photocycles at $40 \mu \mathrm{mol} \mathrm{m}^{-2} \mathrm{~s}^{-1}$ of white light, 650 then transferred to $8 \mathrm{~L}: 16 \mathrm{D}$ photocycles at $80 \mu \mathrm{mol} \mathrm{m}^{-2} \mathrm{~s}^{-1}$ of white light for 6 days. All phase 651 time and period calculations were performed using the MFourfit curve-fitting method using 652 the Biodare2 tool (biodare2.ed.ac.uk, (1)).

653

\section{Cell cycle analysis}

655 For cell cycle analysis cells were pelleted by centrifugation $\left(4000 \mathrm{rpm}, 15\right.$ minutes, $\left.4^{\circ} \mathrm{C}\right)$, 656 fixed in $70 \% \mathrm{EtOH}$ and stored in the dark at $4^{\circ} \mathrm{C}$ until processing. Fixed cells were then 657 washed three times with $1 \times \mathrm{PBS}$, stained with 4',6-diamidino-2-phenylindole (at a final 658 concentration of $1 \mathrm{ng} / \mathrm{ml}$ ) on ice for $30^{\prime}$, then washed and resuspended in 1xPBS. After 659 staining, samples were immediately analyzed with a MACsQuant Analyser flow cytometer 660 (Miltenyi Biotec, Germany). For each sample, 30,000 cells were analysed and G1 and G2 661 proportions were inferred by calculating the 2c and 4c peak areas at $450 \mathrm{~nm}$ (V1-A channel) 662 using the R software. A peak calling method was applied to the resulting histogram, based on 663 a $1^{\text {st }}$ derivative approach (2). The locations of G1 and G2 peaks were first determined using 664 G1 and G2 reference samples and then used to identify G1 and G2 cells in the experimental 665 samples. The area under each peak was used as a proxy for the proportion of cells in each 666 population. 
RNA extraction and gene expression analyses

For qRT-PCR analysis PtRPS and PtTBP were used as reference genes. Each independent replica of the qRT-PCR data was normalized against the maximum expression value of each gene (i.e., gene expression range lies between 0 and 1 across the time series). Average expression and standard error was then calculated and plotted. The full list of oligonucleotides used in this work can be found in Table S4. For the nCounter analysis, gene specific probes (Table S1) were designed and screened against the $P$. tricornutum annotated transcript database (JGI, genome version 2, Phatr2) for potential cross-hybridization. Total RNA extracts (100 ng) from three biological replicates were used for hybridization. Transcript levels were measured using the nCounter analysis system (Nanostring Technologies) at the UCL Nanostring Facility (London, UK) and at the Institut Curie technical platform (Paris, France) as previously described (3). Expression values were first normalized against the internal spike-in controls, then against the geometric mean of the 3 reference genes PtRPS, PtTBP and PtACTIN12.

\section{Selection of rhythmic transcripts and clustering analysis}

For the selection of genes with rhythmic expression in the light-dark cycle, we used microarray data from (4). First, we identified all the genes belonging to the TFs, photoreceptors, cell cycle and metabolism-related categories (pigment synthesis and photosynthesis). Then, transcripts were ranked based on a defined t-value for each time point (mean gene expression of the replica/(1+s.d.)) and those showing t-value $>+0.7$ or $<-0.7$ across the time series, were retained. The nCounter expression data was normalized against the maximum expression value of each gene, in a similar way to qRT-PCR expression data. This normalization was applied to the 3 replicas independently and for each condition (L:D and D:D) with the average expression value used for the clustering analysis. Hierarchical clustering analysis was performed with MeV 4.9 (5) using Pearson correlation. Peak analysis was performed using the MFourfit curve-fitting method defining average expression phases for each cluster (biodare2.ed.ac.uk, (1)).

For the selection of rhythmic transcripts in D:D, expression values were normalized using as reference genes PtRPS, PtTBP and PtACTINI2 and the genes with the highest values of standard deviation from the average expression (M value) over the two time courses (16L:8D and D:D) were selected. A threshold equal to 1 was set using the published P. tricornutum diurnal microarray dataset (4) as background. Gene expression profiles were further empirically examined and false positives eliminated. 


\section{Generation of the PtbHLH1a overexpressing lines}

Transformed cells were tested for the presence of the transgene by PCR and qRT-PCR

703

704 analysis (see Table S4 for oligonucleotide sequences). The full length PtbHLHIa coding sequence was obtained by PCR amplification with the specific oligonucleotides PtbHLH1aDraI-Fw and PtbHLH1a-XhoI-Rv on cDNA template using the Phusion high fidelity DNA polymerase (Thermo Fisher, USA). The PCR fragment was inserted into the pENTR1A vector (Invitrogen, USA) using the DraI/XhoI restriction sites, and recombined with the pDEST-C-HA vector (6).

\section{Data mining, protein sequence and phylogenetic analysis}

The P. tricornutum bHLH1a (Phatr3_J44962) protein sequence was used as the query for blastP analyses on the JGI, NCBI and MMETSP public database (7). Searches of the pfam database for proteins possessing both the HLH and PAS domains were also performed. The retrieved sequences were analyzed using the batch search tool on the CDD (Conserved Domain Database) NCBI server to retrieve proteins presenting at least one HLH and one PAS domain only. We identified 100 HLH-PAS proteins from 71 marine algal species which were aligned using MAFFT (8), along with 22 HLH-PAS proteins from relevant metazoan (Homo sapiens, Mus musculus and Drosophila melanogaster) and unicellular Opistokonta (Monosiga brevicollis and Capsaspora owczarzaki). Preliminary phylogenies were produced with MEGA 7 (9) to eliminate ambiguously aligned sequences, refining the alignment to 107 sequences and a final length of 198 aa ( $<5 \%$ gap per position). The best aminoacidic model to fit the data was estimated with ProtTest 3.4 .2 (10). Phylogenetic analyses were performed with RAxML (1000 bootstraps) and MrBayes 3.2.6 (2.5 million generations, 2 runs, 25\% burn-in) on the CIPRESS gateway (11). The final tree was edited in FigureTree 1.4 (http://tree.bio.ed.ac.uk/software/Figure tree/). GenBank accession codes of the genes utilized in the bHLH-PAS phylogenetic analysis are reported in Table S3. 
$731 \quad$ Figure S1 - Annunziata et al.

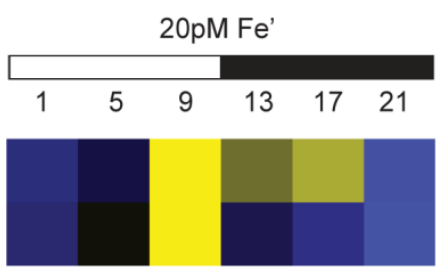

732

733

734 Fig. S1. Diel expression patterns of PtbHLH-PAS genes under Fe-depletion conditions in

735 12L:12D photoperiods. Diel expression patterns of PtbHLHla and bHLHIb in normal (400

$736 \mathrm{pM} \mathrm{Fe}$ ') and iron depletion conditions (40 and $20 \mathrm{pM} \mathrm{Fe')} \mathrm{were} \mathrm{obtained} \mathrm{using} \mathrm{transcriptome}$ 737 data extracted from (36). Light and dark periods are represented by white and black 738 rectangles. Expression values are given relative to the maximum expression for each gene, 739 where ' 1 ' represents the highest expression value of the time series.
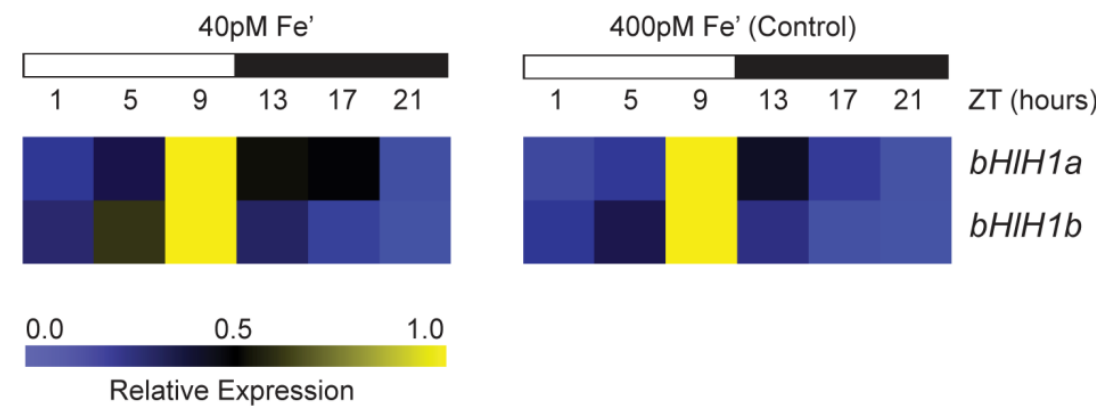

740 
Figure S2 - Annunziata et al.

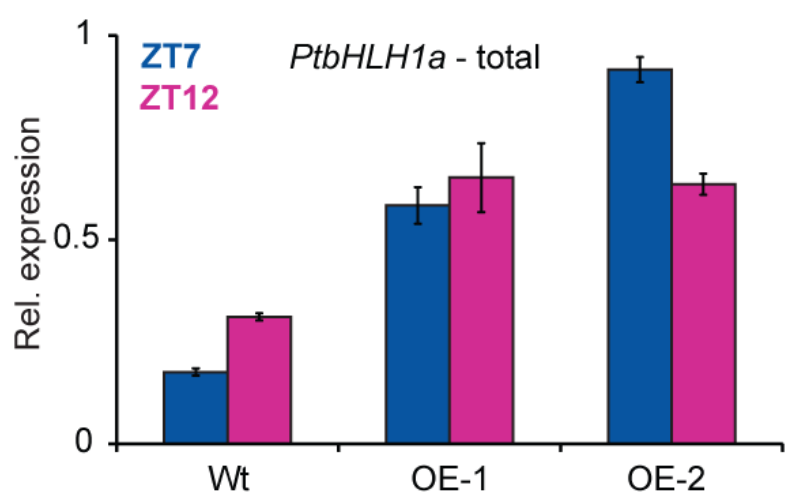

746 Fig. S2. The Lhcf2p:bHLH1a-3xHA:Lhcf1t construct drives over-expression and 747 anticipation of PtbHLH1a. Quantification of total PtbHLHIa transcripts in two independent 748 PtbHLH1a over-expressing lines (OE-1, OE-2) compared to the wild type (Wt) strain. Cells 749 were grown in 16L:8D photocycles and sampled at the ZT7 and ZT12 time points. qRT-PCR 750 expression values were normalized using PtRPS and PtTBP as reference genes and represent 751 the average of two biological replicates $(n=2) \pm$ s.e.m (black bars). 

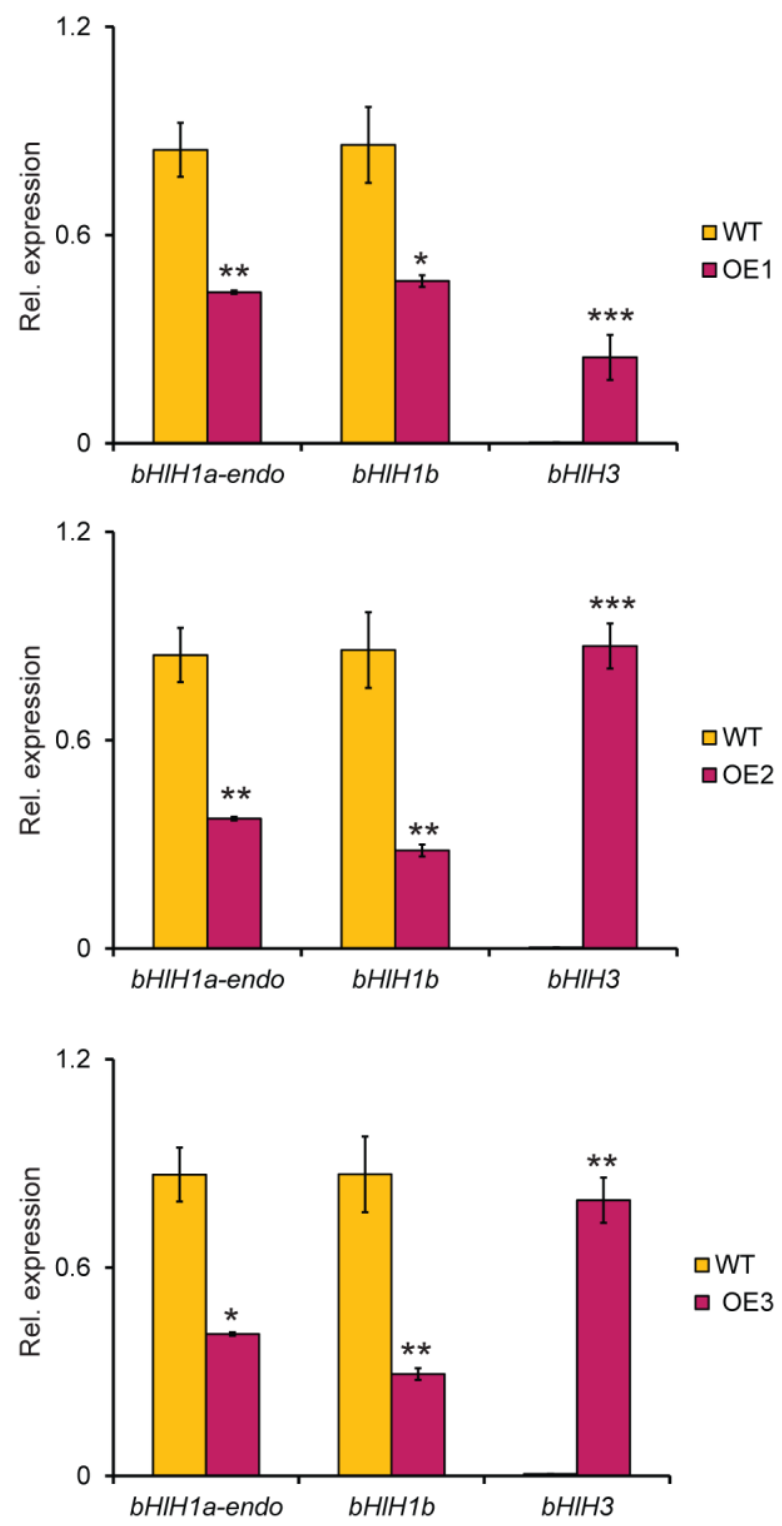

757 Fig. S3. PtbHLH1a over-expression alters rhythmic diel gene expression in three independent lines. Quantitative gene expression analysis by qRT-PCR of PtbHLHIaendogenous, PtbHLH1b and PtbHLH3, transcripts in 16L:8D entrained Wt, OE-1, OE-2 and OE-3 cultures. Samples were harvested at ZT10. PtbHLH1a-endo refers to the transcript levels of the endogenous gene only. Expression values represent the average of 3 biological replicates \pm s.e.m. (black bars) and were normalized using PtRPS and PtTBP as reference genes. For each gene, expression values are represented as relative to its maximum expression corresponding in the graph to ' 1 '. $* \mathrm{P}<0.05, * * \mathrm{P}<0.01, * * * \mathrm{P}<0.001$, t-test. 
Figure S4 - Annunziata et al.

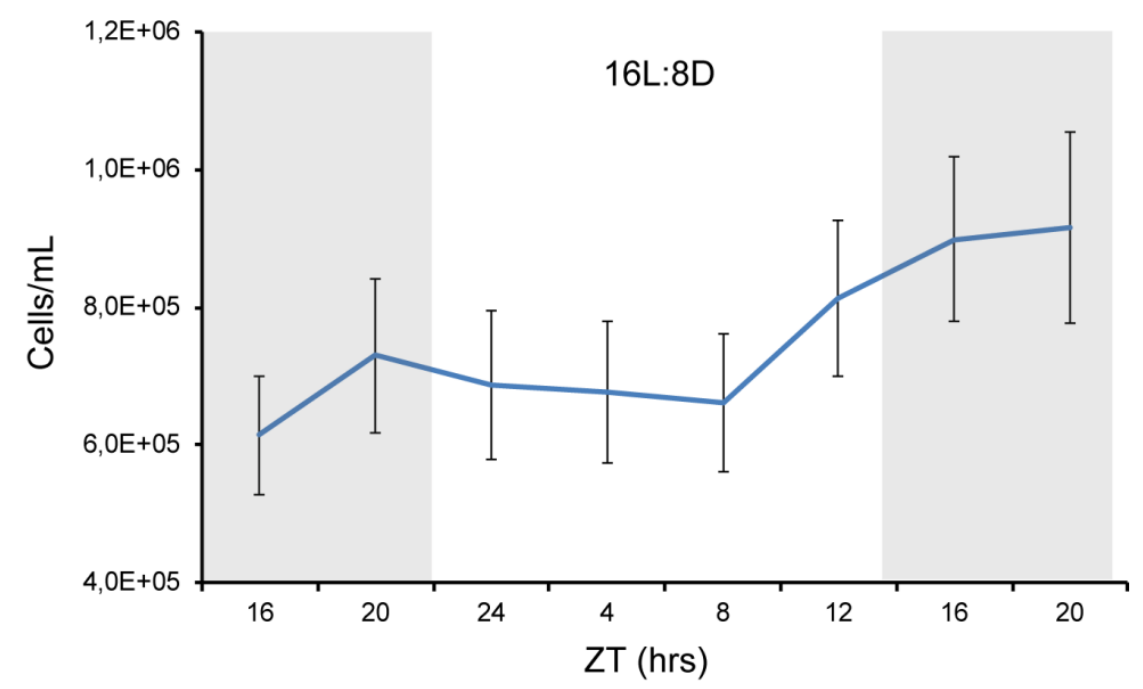

769 Fig. S4. Diurnal cell growth dynamics in $\boldsymbol{P}$. tricornutum. Diel cell number measurements in

770 wild type cultures grown under 16L:8D photoperiods. Values represent the mean counts of 771 three independent biological replicates \pm s.e.m. (black bars). 
773 Figure S5 - Annunziata et al.

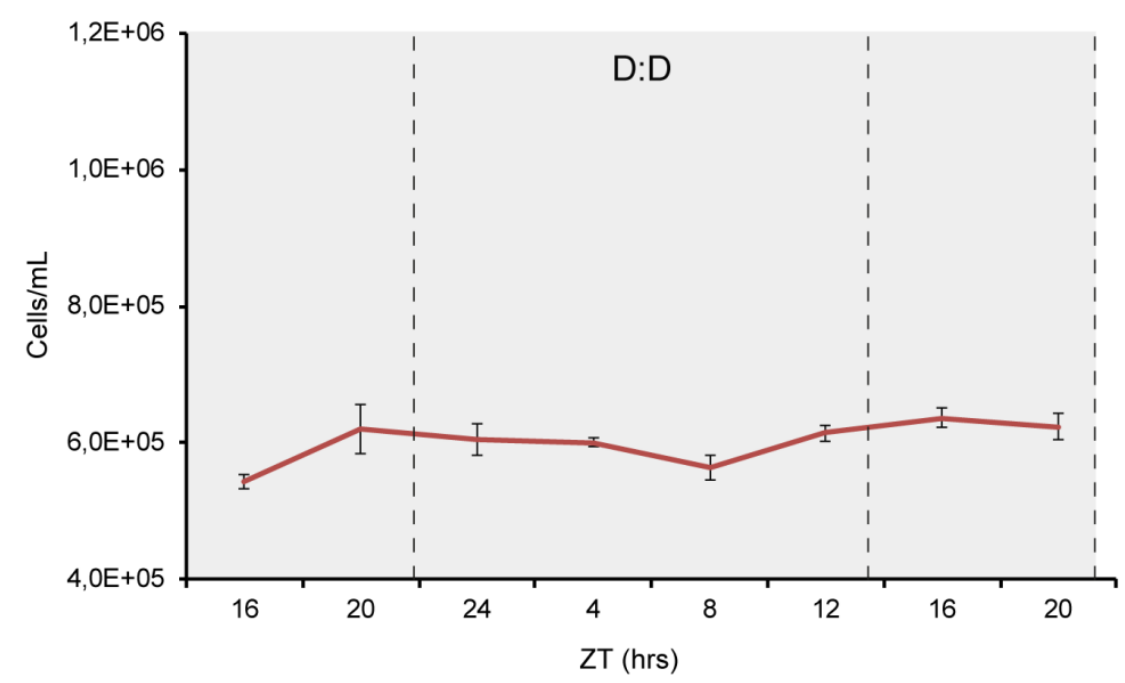

775 Fig. S5. Cell division is arrested in continuous dark conditions in P. tricornutum. Diurnal

776 cell growth measurements of the wild type cultures entrained in 16L:8D and then transferred 777 to continuous darkness condition (D:D). Values represent the mean counts of three 778 independent experiments \pm s.e.m. (black bars). 

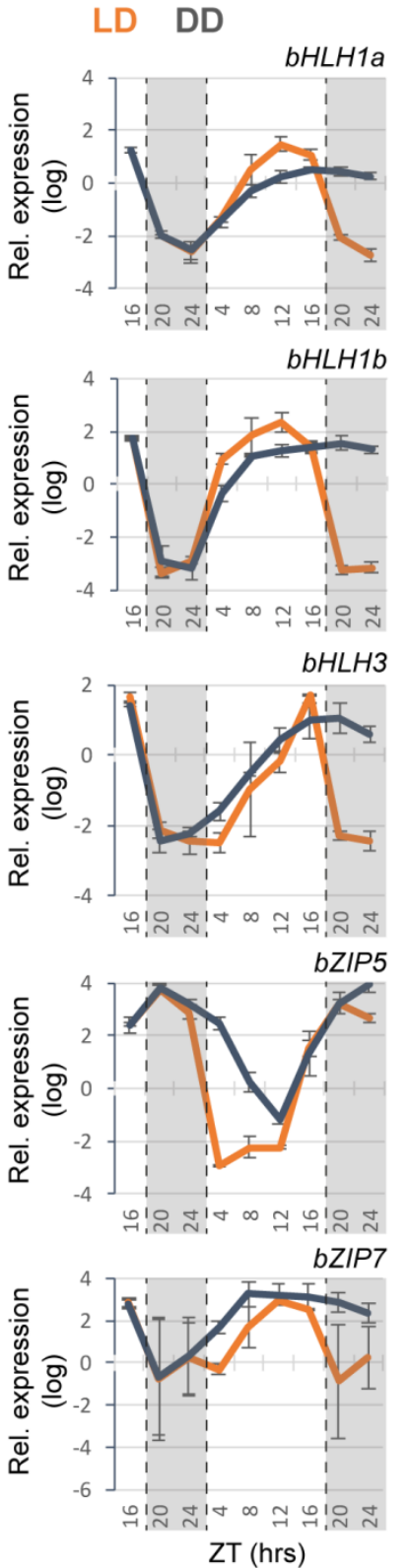

782 in grey $(\mathrm{D}: \mathrm{D})$.
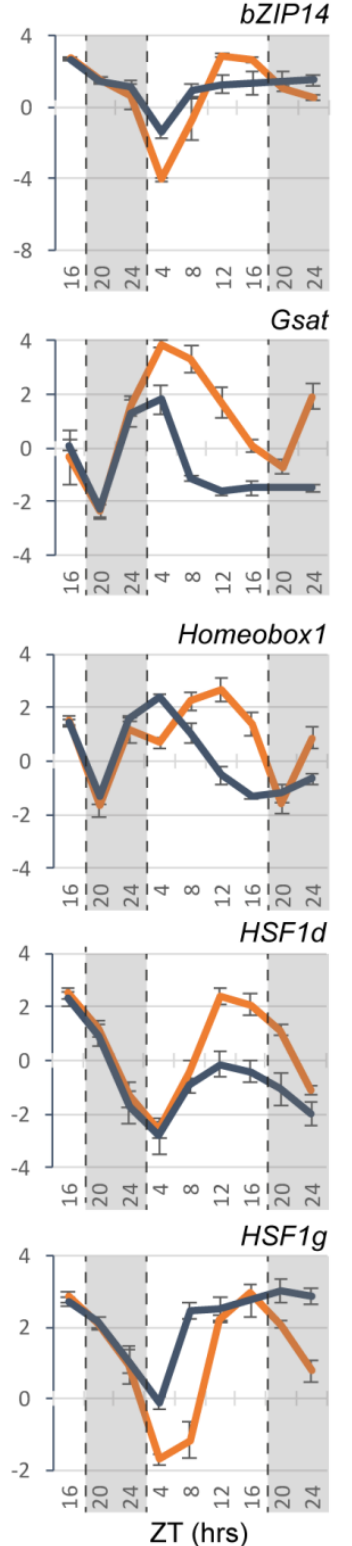

HSF3-2e

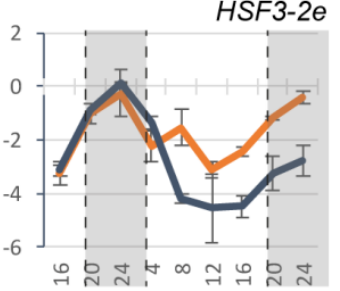

HSF3-3a

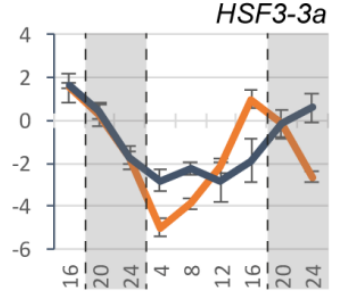

HSF4-7b
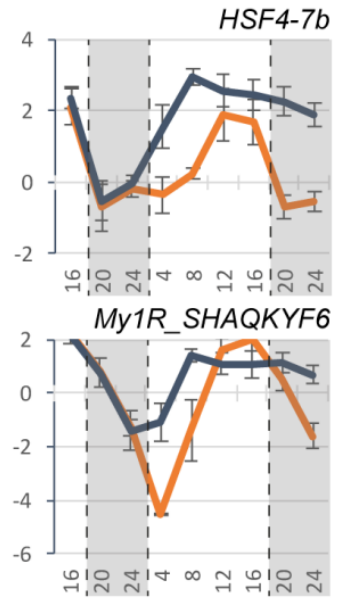

Myb2R3a

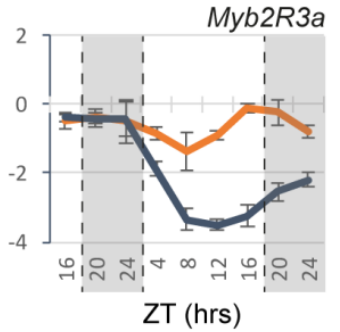

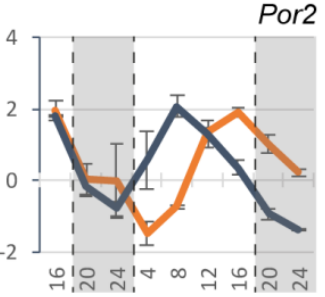

Taz2
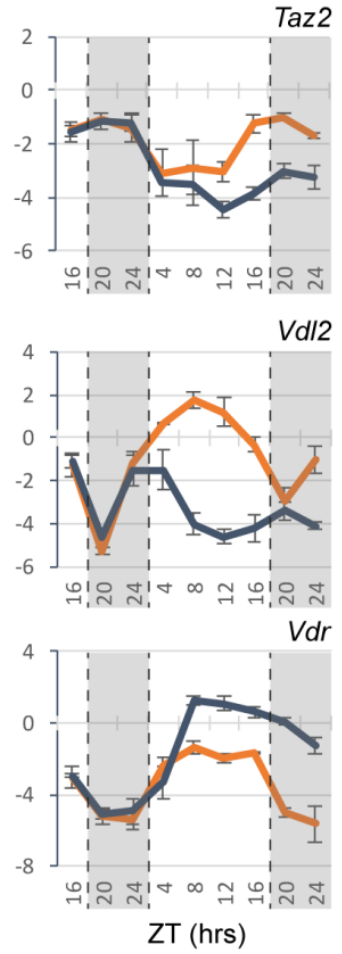

Fig. S6. nCounter expression analysis of genes maintaining rhythmic expression in D:D conditions and 16L:8D condition in Wt cells. Data represent the average expression of biological triplicates $\pm \mathrm{SD}$ and are normalized using the PtRPS, PtTBP and PtACTIN12 reference genes. For each gene, the expression value is relative to its maximum expression corresponding in the graph to ' 1 '. Results for cells grown in $16 \mathrm{~L}: 8 \mathrm{D}$ cycle are shown in orange (L:D); Results for cells in constant darkness (following 16L:8D adaptation) are shown 

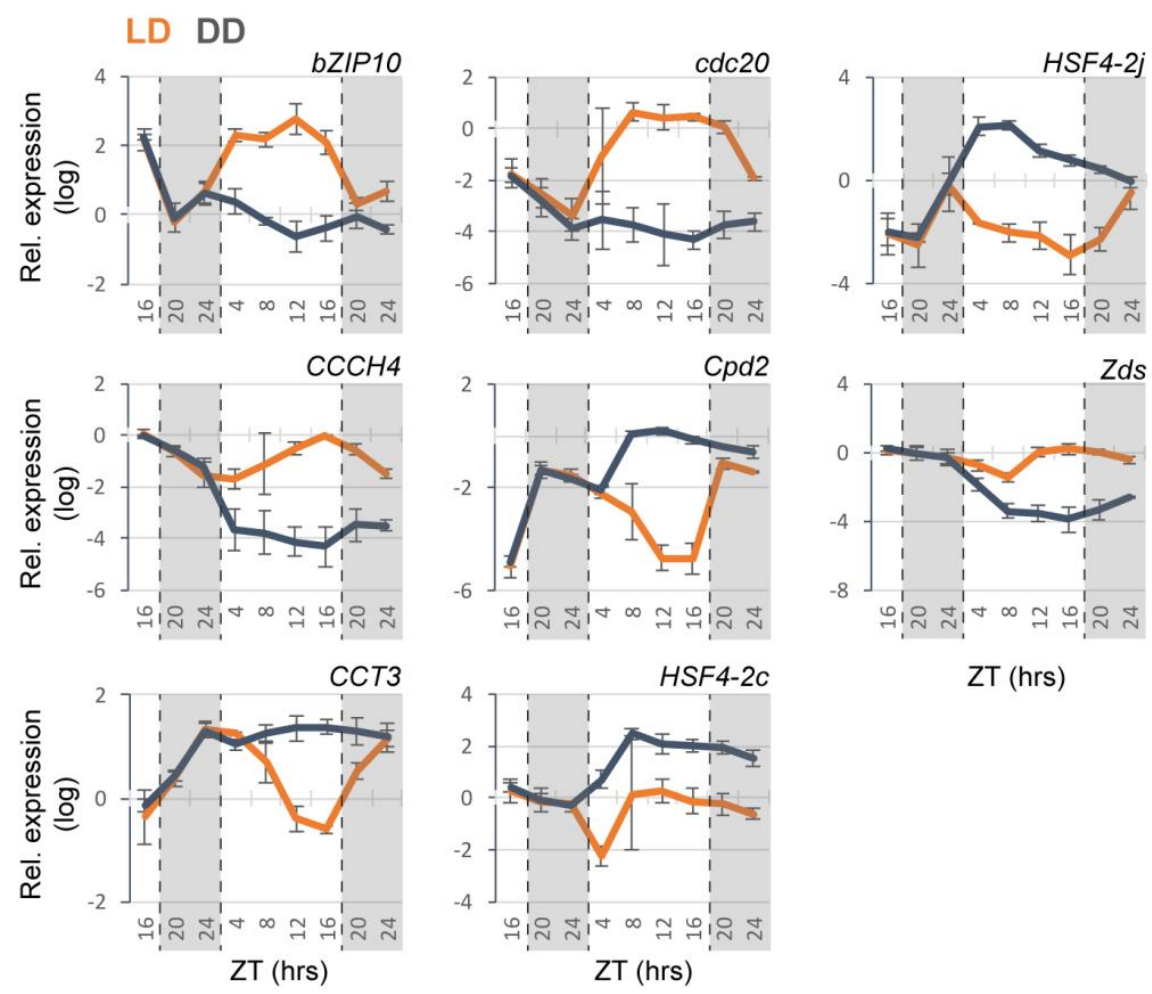

ZT (hrs)

Fig. S7. nCounter expression analysis of selected genes with altered rhythmic expression in Wt cells in D:D conditions compared to 16L:8D condition. Expression values represent the average of three biological triplicates $\pm \mathrm{SD}$ and are normalized using the PtRPS, PtTBP and PtACTIN12 reference genes. For each gene, the expression value is relative to its maximum expression corresponding in the graph to ' 1 '. Results for cells grown in 16L:8D cycle are shown in orange (L:D); Results for cells in constant darkness (following 16L:8D adaptation) are shown in grey (D:D). 
809

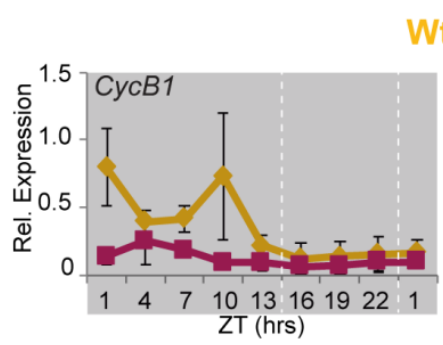

Wt OE1
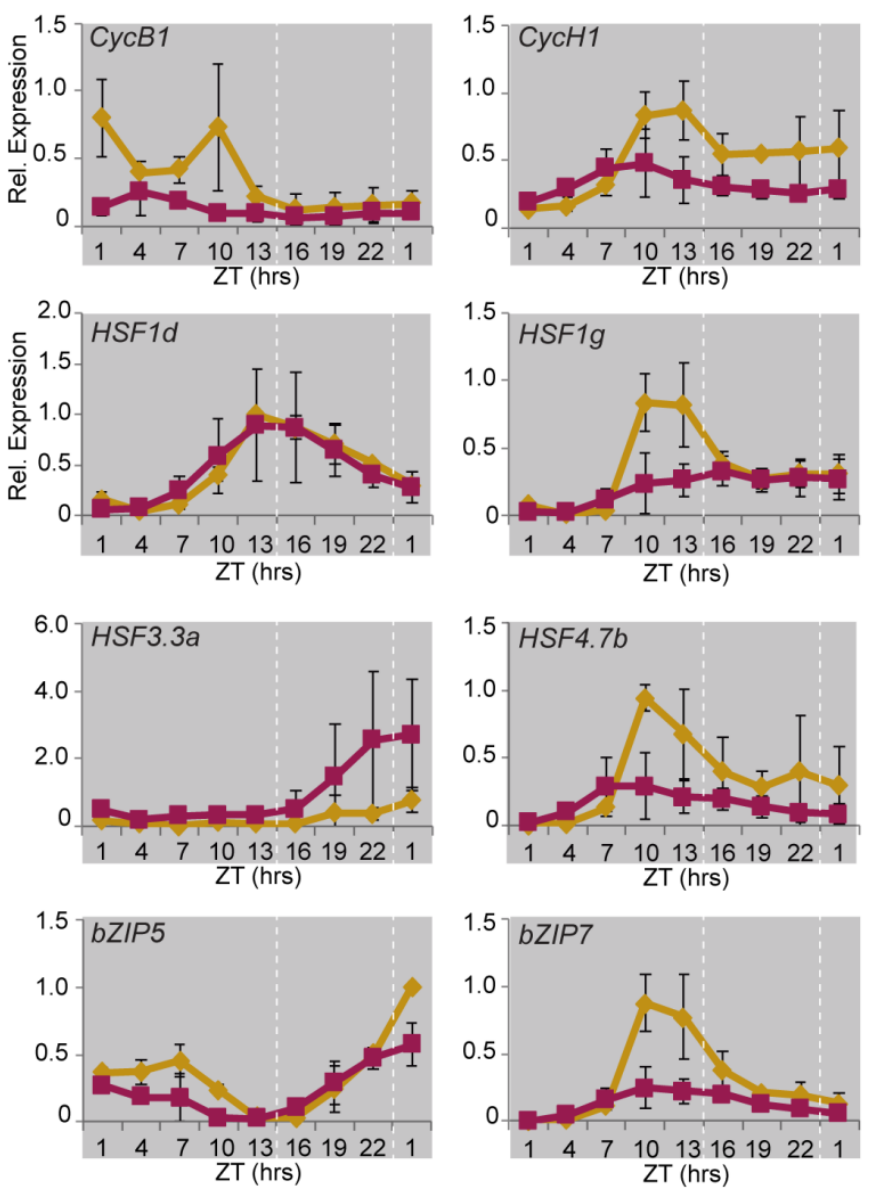

812 Fig. S8. nCounter analysis of selected rhythmic gene expression profiles in continuous

813 darkness in Wt and PtbHLH1a OE-1 cells. Cells were entrained in 16L:8D cycles, then

814 transferred to D:D and collected every 3 hours for $24 \mathrm{~h}$. Expression values represent the 815 average of three biological replicates $(n=3) \pm$ s.e.m (black bars) and have been normalized 816 using PtRPS and PtTBP. 


\section{Table S1: Accession codes and sequence probes used in the nCounter analysis.}

\begin{tabular}{|c|c|c|c|c|}
\hline Category & Gene Name & $\underline{\text { NCBI Accession }}$ & Phatr3 Accession & $\underline{\text { nCounter Probe Target Sequence }}$ \\
\hline Reference Gene & Actin12 & XM_002182185.1 & Phatr3_J29136 & ATGTAAGCCTGCAGCCACTGAGGACTTGTGCTCGTAACCCTGATTTCGATATTCCAAAATGCGGCTCACTAAAAAGACATCGTAGTCCAGTGCCAGTCCC \\
\hline Photoreceptor & Aureo1b & $\# N / A$ & Phatr3_J49458 & TTATTGCCGAGTGCTGTACCTCGAGTCCGTTGTTTGAAGAAATGGACGGCATAGATCAAACGAAAGGGGCCAATCTGGAACGTGCCGATTTTTTCATTGAT \\
\hline Photoreceptor & Aureo2 & XM_002183279.1 & Phatr3_J15468 & AGACGTTACTGTCACTCTCATGAACTACACTGCCGATGGTACACCATTTTGGAACAAGCTCTTTATTGCCGCATTGCGTGACGCGCAAAATAACATTGTC \\
\hline TF & bHLH1a_PAS & XM_002179032.1 & Phatr3_J44962 & TACTTTGGTCAATATCAAAGTCAGTTTGGTACGAACAGCTCAGCATAGCCCTCGGTTTTTCAATGTGGCGTTGGTGCCATCAGACGATGCAGCGAAGCTG \\
\hline TF & bHLH1b_PAS & XM_002178812.1 & Phatr3_J44963 & AGGAATTGACTACCGTGCTGTTTTCAATCATTGTCCATATGCCATGGGGGTGGCTTCATTGGACGGAAGAATACTGGTCTGCAACAGCTCTTTCGAATCT \\
\hline TF & bнLнз & XM_002176560.1 & Phatr3_J42586 & ATCACTAGCGACACATTCAGATTCATCTGCGGGAGAAGGACGGTCTATTCCGTCAGTAGTTGGTACAAGTGTTAGCGGTTCAAGTCGCTCTGGGAACTGC \\
\hline TF & bHLH5 & XM_002177858.1 & Phatr3_J43365 & ACCCTCTTGCAGCGCAATCATTCGCTCCCGCATACGACTATGGGTCGATTTCTCAATGAACGCCATTCCTTTTCCTCAGACCTTTCGCTCTCGCTGGCGT \\
\hline Cell cycle & BUB1/MAD3 & XM_002178639.1 & Phatr3_J10954 & CGTGTGCTGCATGTATGCGGACAAAACCGATCGTCCACTGGAAGTTTTCCAGCATCTACATCAGCAAAGGATCGGGAGCGATATTGCCGTGTTCTGGATG \\
\hline TF & bZIP10 & XM_002177776.1 & Phatr3_J43744 & AAGCTGCTCGGGAATCGCGTCGGCGGAAGAAGGTTATGATTGAAGAACTTCAACGCAGCGTGATTTTCTTCTCGCGTGCCAACGGAACCCTCAAACAACA \\
\hline TF & bzIP13 & XM_002181635.1 & Phatr3_J47278 & CGCGGCGGCTTCGCGTGCTAAGATTCGATGCCGCATCACCGAACTTGAAACCGAGGTTAGTGGTTGGAAGGATAAATACACACAAGCAATGGAACGCTTG \\
\hline TF & bZIP14 & XM_002179477.1 & Phatr3_EG02108 & ATGCAGAGCTTGGAGGGTCGAGCTACGGATCTCAAGCATGAGCAAATTCGTTTAAAACAGATCATTAACGAAAAGAACACGGCGAACATTCTGGTGGGGC \\
\hline TF & bZIP16 & XM_002181462.1 & Phatr3_J47279 & СтTTTGGAAGCAGTCACAAATCCGCAAACATGAACAGTTCCAACGCCCAGGAAGTCGCTCGTCAACTGAACGAAGATCCTGCGCTCGCTCGGAAGCGTCG \\
\hline TF & bzIP18 & XM_002176556.1 & Phatr3_J42577 & АСTCTTCTATAGCGGAATCCGAGGCCGATTTCAAAACTATAGCTCAGGCTGCTGTTTCGAATCTGATCATGTCGGCTGGAACTACCAAGGTTGAATCTGG \\
\hline TF & bZIP19 & XM_002183101.1 & Phatr3_J48701 & ССTGCACCTTCTCAAATCAGCTGTGCAACGAGTGCAACAAGAAGAAATATTGGCTGCCGAGGCATTACCTACACCTTTTATAGGCGTTTTGCGATTTGGC \\
\hline TF & bzIP24 & XM_002184633.1 & Phatr3_J49887 & TTGTCACGGGGCCAACAGACTATCTGGAAAGATCACTTCTTGCCCAAAGAGAAGCGGAAAGAACTCGTTCGGCTTTGTCAAGCATCGCGCTTCGGGGGAA \\
\hline TF & bZIP25 & XM_002185584.1 & Phatr3_J46647 & TCGTCGTTCACGATGGCATCTGTACCAACTCACCTAGTAGGAAATACTGTCATCACTGATACCAGTTATCAGCCAGTTTCGTCGCTATTACCTGCAGCGG \\
\hline TF & bZIP26 & XM_002182641.1 & Phatr3_EG02494 & GATTCTTTGACGGACTCAAGGAGCGACAACTTGTCATCGACATCTCAGGAGCTTTCGGTGCGACAGGAAAAGGTCGAAGCCGCTTTGAAATCGAAACCTC \\
\hline TF & bZIP5_PAS & XM_002179145.1 & Phatr3_J45142 & СCCTTGTTTCGAACAGCGCATTCGATCCGCAAAACAACGCTAGCAACAACGCACAATCACAGTCGCAAATGATGATGCAGAACGTGGCTATTATGAGTG \\
\hline TF & bZIP6_PAS & XM_002184884.1 & Phatr3_J50039 & CGCGCAATCTAATACACAATATCAGCTAGACAGCCCACAACATCCCGAAAATACGATGTCGCAGTGTAGACCGCAGCAGGGTTCAAATAATTCGGCGAAC \\
\hline TF & bZIP7_PAS & XM_002183297.1 & Phatr3_J48800 & CGTGAGAGACCTTGCCATGCGTAATCAAGGATTAGAGGCATTAGCGGCGATTGCGACAGCAAGCTCGTCTTCAGGGCACACGTTTAGTACCAACACGAAT \\
\hline Metabolism & CaThioredoxin & XM_002184396.1 & Phatr3_J49634 & CTGGAGAGCCCTACCGTGCTAGTGTCCGTGTATTGGAAAAATGTCGTTGTTTTGCGTTCAGCGTAGAAGACATTCCTGACAGCTCCATTCTGAGTCTGAA \\
\hline TF & $\mathrm{CCCH} 4$ & XM_002181104.1 & Phatr3_J13664 & CATGTGCCGCCTTGGAGCGTCGCACATTAACATGTCTACTGGCGAAAACATTCGAAAGGACATTGATGGTCCGAAGCCCAAGCCAATCTTAAATACTCTA \\
\hline TF & $\mathrm{CCCH} 8$ & XM_002186091.1 & Phatr3_J44042 & TCCGAAAAATATGTGCGAAGAGTCCCACTTGTCGCGTATGTTCTTGGCTCATACGAGTATTGCGCCGACGGAAGTGGAAGAGATTGAGTTTGCGGGTGAA \\
\hline TF & $\mathrm{CCHH}_{1} \mathrm{CCCH} 2 \mathrm{O}$ & XM_002179821.1 & Phatr3_EG02317 & TAACTACAACAACGTTCCTCAGCTAAACGGTTTGAACCATCTGCCTTCTAAGAACCTATCCGACTTACATTCCCACTATCAAGATCATAGGAGTCCAAAC \\
\hline TF & СCHH14 & $\# N / A$ & Phatr3_J34600 & GCCCAGTTTCTGCGGAGGCATCCCGTTCAATCCGCCGATCTGAGTTACGAAACGCACTACATGGCGTACCAAAAAGTTTACACTGATGCCCGAGTTGTTC \\
\hline TF & ССТ3 & XM_002185983.1 & Phatr3_J43850 & AAACGCTGCCGCCGCGTTTGGAATAAGAAGATTCGCTACGGTTGCCGTAAAAATTTAGCAGACCGCCGGTTGCGCGTGAAGGGGCGATTCGTGAAACGTT \\
\hline TF & СCT4 & XM_002178133.1 & Phatr3_J44285 & TATGAATGCAGTCGACCGGGAATTGAGTTTCCATCAAACAACAGAGCTAAGCTGGTCGAGCACCAGCACGGCCTTTCGCCCATTCCTTTTCACGATAGTG \\
\hline Cell cycle & cdc20 & XM_002180546.1 & Phatr3_J12783 & TTACGACGGAATTGCTTTGAAGAAAATCAGAACACTTCATGGGCACACAGGCCGAATATCGTCGTTGGGATGGAACCAACACTGGTTGAGTTCAGGCGCA \\
\hline Photoreceptor & Cpd2 2 & XM_002179343.1 & Phatr3_J54342 & TCTGGTGTGCGGCAATACGGCGGACAGTGTTTCGGAGCTCTGTAAAATTGCTTCTGAGATTGGCGCGAGTGCAGTCTATTGGAATCGCGAGATGACACCT \\
\hline Photoreceptor & Cpd3 & XM_002180035.1 & Phatr3_J51952 & СTCTGATTTTTCTCCGATACGCGAATACCGTCAATGGATGGAACTCCAGGCCGTACCTATCTTGGAGGAAGCGAAGGTCCCGTTTTATCAGGTTGATGCC \\
\hline Photoreceptor & Cpf1 & XM_002180059.1 & Phatr3_J27429 & GGATGCGTGCCAAAAATCCGAATCACTCCTTCCGATTTACGTAGTCGACCCTGAATTTCCCTTCGCGCAAACTGCTGGGTGCCGCGCCGGTACAATTCGT \\
\hline Photoreceptor & $\mathrm{Cpf4}$ & XM_002184521.1 & Phatr3_J55091 & СTCGATCCCTTGTTGCGGCGCAAACGAGACTGCATTTCCGTAATGGGTCTACCTAATGATTTTGTAGACTCCATTGTCGAGGCAGCGTTTGAAGCAGCTG \\
\hline Photoreceptor & CryP-like & XM_002178853.1 & Phatr3_J34592 & CACTTTATGGTGGCGAAACAGCCGGACTCGCACGATTGTTTGAAGGATTACTTCGAAACGCGCAACGGGATGCTGGGGCCGAACTATTCGACCAAATTCA \\
\hline TF & CSF2 & XM_002185522.1 & Phatr3_J41601 & GAACAGAACGAAAAGATTCGGGACGCTTATGCATTCCAACAGACGAAAATCGACGAGGCTCATCAACTTATTGTCAATTTGGGCATGGACGTTGCAGACT \\
\hline Cell cycle & CYCB1 & XM_002180361.1 & Phatr3_J46095 & TTAGAGAAAAGTCAATCGCAAATCCTGAGCTCCGAGCGGTGAATAAAAAGTACAGCGGTCATCGATACGGCGGAGTTGCTTCGACCGTTCTGGTATTTGA \\
\hline Cell cycle & CYCH1 & XM_002185709.1 & Phatr3_J36892 & TTTGCGATGGCTCGCGTTTGACGTTTCGGTTTCCCATCCGCACCGAGGAGTTGCCGCAATCGTCAACCATCTCGTGGATTGCTCACCTCAATGGTTTGGC \\
\hline Photoreceptor & Dph & XM_002179026.1 & Phatr3_J54330 & CAACAATTCCATTACAACCAAAGAGCTGACGGAATGTGATCGTGAGCCTGTGCACTTGATCGCAAACGTACAAGGGGGTACCGGCCATTTGTTGTTCATT \\
\hline Cell cycle & dsCYC2 & XM_002179247.1 & Phatr3_J34956 & GATATTGCCGTTACCGAACGCGAAATAATGACTGCTCTTTCCTGGCACTTGCATCCTCCAACCGCTATTGGCTTTTCACGAATGTACTGGTCTCTGCTGG \\
\hline TF & E2F_Dp2b & XM_002181452.1 & Phatr3_EG02016 & TGATTTGAATAGAGCTGTGCAGGAAATGAGGGTACAAAAGCGCCGGATTTATGACATTACCAACGTTTTGGAAGGTATTGGGCTGATTACCAAGGATAGC \\
\hline Cell cycle & E2F1 & XM_002176842.1 & Phatr3_J43065 & GTGTGGGTCTCATCGAAAAACGATCCAAAAACACAGTCGCTTGGAAAGGGAGTGAGCTTCTTCTTGGCTCGTCCTTTTCAAGTGCCGCCAAGCAGAGAAT \\
\hline Cell cycle & Ftsz & XM_002185088.1 & Phatr3_J42361 & GAAAAAGGAGAATGAAGCAAGCGATTGCCGCGATTGAGAGGTTGCGAGAGAATGTGGATACGGTCATCGTAGTGTCCAACGACAGACTGCTCGAGATTAT \\
\hline Metabolism & GapC1 & XM_002182255.1 & Phatr3_J22122 & TGAAGGGATTCCTCGGATACTCCGACGAACCGTTGGTCTCCACCGACTTTGAAGGTGACTTGCGCTCCTCCATCTTTGATGCCGATGCCGGTATCATGCT \\
\hline
\end{tabular}


bioRxiv preprint doi: https://doi.org/10.1101/271445; this version posted May 26, 2018. The copyright holder for this preprint (which was not certified by peer review) is the author/funder. All rights reserved. No reuse allowed without permission.

\begin{tabular}{|c|c|c|c|c|}
\hline Metabolism & Gsat & XM_002180931.1 & Phatr3_J36347 & ATTCGTTTGGTCCGCGCACACACGGGTCGGGAAAAGGTCATCAAGTTCGAAGGATGCTACCACGGACACGCCGATTCATTTTTGGTCCAAGCCGGATCCG \\
\hline TF & Hox1 & XM_002184935.1 & Phatr3_EG02213 & AACAAACATCGGTTCACATACTGCGTCATACCGGACAAGTCCCGACAGTCGAAGATGTTACGATCCTTACCAATGTTCCGGCGGAACGCATTCTCCGAAC \\
\hline TF & HSF1.3b & XM_002179894.1 & Phatr3_J35419 & СTCCAGGGAACAACTCGATACCAGAGTTCCTCTATCAGCTAACAAAGATGCTGACGGATAACAACCGAGATATTATTGAATGGACAAATGGCAAGATTGA \\
\hline $\mathrm{TF}$ & HSF1a & XM_002177362.1 & Phatr3_J43051 & CCACCAACGTCGGATAGCTCCGACTGGGGTTCTCCAATCCAATCATCCAAGCCAGCTGTTGATCTCTTAACGAGGAGTATGCCGCAATGGACAAATATAC \\
\hline TF & HSF1b & XM_002181395.1 & Phatr3_J47181 & TTGTAGTGGACACTTCGATGCTCGAAGAGGTCATCAACGAAACGCAGCCACTCGTTGGAGACAAAGGTAGCTTCTCCCTCTTGACAAATAGAGCACCAGT \\
\hline TF & HSF1d & XM_002178673.1 & Phatr3_J44750 & GTCACCATTCCAGCATCGGATGCGGCTCCTTACGCCATTTCTCCCGAAACCTCACCGAGAGACTTGACTCTGAGACACAACGAAATTCCCAACCTTGATG \\
\hline TF & HSF1g & XM_002177016.1 & Phatr3_J42514 & ATAGAGAATCTGCAAGCACTAGGGACCATGACGACTACTTCATCGAAGCGTAGCATTACAGAGAGCGAAGATAGTTCCCTGTCAGACTCAGCATCAGGAG \\
\hline TF & HSF2.1a & XM_002181681.1 & Phatr3_J47360 & AGCCCTTGCCGCTGGAATTTTGGGCTCGTTGTCGCCTCAAGTCAACCAACCACAGCCGTGGATGATCCAACACGCCCTGTCAATCGCTCAAGCGTTAGTT \\
\hline TF & $\mathrm{HSF} 2.2 \mathrm{c}$ & XM_002185405.1 & Phatr3_J50481 & GGACTGGAAACTGTAAGCGCGACATATTTCCGCAGCGCCTTCTAGCGATTTTGAGCGAATCATCCCTATCTGACATTATCACTTGGCTTCCACATGGACG \\
\hline TF & HSF3.2b & XM_002186124.1 & Phatr3_J44099 & TTGACTCCTCGCGACCAGCAAGCACTTAGCGCGTTTCAACAGTCCCTCGGTGCATCCGAGAGTCAGTTCAAGTCTATGAGCTTTTCCACCACAACTCCTC \\
\hline TF & HSF3.2e & XM_002179404.1 & Phatr3_J45206 & TTGGAACGGGAACGCTTTCCCTCAGATTAGCAATCCGCAACCTTCGCCTACTCCTCCAAGTTCGACGATGGTAAATACTTCGGACTTAGCAAGACTACTT \\
\hline TF & HSF3.3a & XM_002179738.1 & Phatr3_J45393 & TCTGGGCAAATCTTTCGTATGGAAGGTTTGGCGACGCCGAGTAGTCAGGGAGTTGGCGTAACCACAGGCAGTTTGCCGCTTCGAGACCAAGACGCTGTTA \\
\hline TF & HSF3.3f & XM_002179735.1 & Phatr3_J45389 & CATGCCAATTGAACACCCAATTCAGCCATACATGGAAGAGCTTAAGAGGGTCCATCTTGCAGCGGCGGCGGGAAGTCTCGAAAATGCGCTTCGAATTTTG \\
\hline TF & HSF4.2c & XM_002182762.1 & Phatr3_J48361 & CGCACGTATATCACGATTTCGCACAAGTGCGGCCCGACGCGGATCCCATGATGATCGTCCGGAAAAAGACTGGAGGAGTCACTCAGCCATTTCCCGAAAA \\
\hline TF & HSF4.2j & XM_002177548.1 & Phatr3_J43363 & GTCGGCGGACGGGCCTTTATCATTCACAAGCCTGACAAGTTCTTTAAGGATATAGTGCCGCTATATTTTCGCCAGTCGCGGCTGAGTTCATTCAAGCGGC \\
\hline TF & HSF4.3a & XM_002184368.1 & Phatr3_J49594 & CGCGAACACTCGCGTTCCAATGTCGCGGAAGAACCTTCCAAGCGAACCTTTGTTCAGCATATTTACCATGACCATATGAACGACCCTGAGCAGGTGGATG \\
\hline TF & HSF4.3b & XM_002183012.1 & Phatr3_J48558 & CTGTCTTGAGCCTATCGCAGCAGTGGCGCCGAATTTCTACGCTATGCCAACTACTGAAGGTGGAGACAAAGACCAATTATTCTCGATCCAAAATACGTGG \\
\hline TF & HSF4.3c & XM_002183081.1 & Phatr3_J48667 & AAACTGTCAGTCATGCTTGACCATGTAAAAGCCGCAGGATTGGACGACGTTATTTCTTGGGCCAGTCATGGCCGCTGTTTTAGCATTCACAACCCAGACC \\
\hline TF & HSF4.4b & XM_002184371.1 & Phatr3_J55070 & CCCATTCCGGTAAACCCGGTTTCCAACTTGGGCGTATCCAGAGCGCCTAGTTTTGGTAAGCTCGAACCAGGCCAATTCGCTGCTTATGGCGGTTGTATGA \\
\hline TF & HSF4.6a & XM_002184347.1 & Phatr3_J49557 & CTCAAACACTGGCGATTGCCGTATCGCCTCGTTCCGCCATCCAGTGCACGCCAACATCGTGAATCCTTCGCACTTTCGCTCTGTCTCCAATAACGGGCAC \\
\hline TF & HSF4.7a & XM_002185271.1 & Phatr3_EG00092 & CGGACATCCCGGGGCGGATTTGAGATCGACGCGATAGTGTCATTCTTGCGTTCCAAGAGTATTTCGCAACTCTACATTATCGGTGGTGACGGTACTCACC \\
\hline TF & HSF4.7b & XM_002184277.1 & Phatr3_J49596 & CTCGAGGTGGTGTGGGAACTCCTTTTCCACTCAAGCTGCACGAAATGCTTCAAAATGTCGTACAAGACGGCTACGCTCACATTGTATCGTGGCAGCCTCA \\
\hline Metabolism & Lhcx1 & XM_002179724.1 & Phatr3_J27278 & AATCCTTGAGAATCTTCAGGGTTAAAGAGTGCATCCATCCAAAACTGGAGGTATGGCTGGTCACATGCACAGAGTTAACAACCAATCCCTTTTCAAGCGA \\
\hline TF & MYb1R_SHAQKYF4 & XM_002178425.1 & Phatr3_J44331 & CGTATCTGCCGAATCGGTCTCTGGTGCAAATCAAGTCCCACGCCCAAAAAGTGCTCAAGCGCATCGATCAAGGCGAACACGTCTTCCGACGTATCGAGGA \\
\hline TF & MYb1R_SHAQKYF5 & XM_002181623.1 & Phatr3_J47256 & ACGCTTCACCTGGCCCGAAGACTTACACCGGGATTT'TGTATCGGCAATTTTTGATGTCGGCTTGAAACAGTCGTCACCGTCCACCATTTTGGAAACCATG \\
\hline TF & MYb1R_SHAQKYFG & XM_002180835.1 & Phatr3_J46535 & TAACGACGAAGCGCAAATCCTTACCGACAAACT TGCCGTCCCGCAAAAAGGGTAAGACACGAAAGTCTGCCGCCTTGGTACAGCGTAATACTTCAGTTTC \\
\hline TF & Myb1R10 & XM_002181361.1 & Phatr3_J37257 & CATATCCCTCTTCGGGGCCCTCACTTCCCTGTACAAGTGAATCTTTGGGGGTGAAAGCGAATCGTAGAAAGAAACGTGCTAAGGACGAGACCTCGGTTCT \\
\hline TF & Myb1R4 & XM_002181410.1 & Phatr3_J47205 & AAAGTTCGTTGCAGGTTTAGACGGAACGTCTACCGCATTGTCAATGGGAACAATCCGGCGAGTAGGATTCACCCATATGCGATTGAACTTCTGGAAGCCG \\
\hline TF & Myb2R3a & XM_002181911.1 & Phatr3_J47726 & TCCATCTTTCCTTTGAGCGACTGTGACGACCTGTGGAGGGAGAGTGATTTTTGTCACTGTGAAGTTGTCGGTACTCCGGCACTCGGCAGGTAAAATACTC \\
\hline TF & Myb2R3b & XM_002182076.1 & Phatr3_EG02275 & GCGCACAACGGTTTAAAGTGATTCGGGATTCATTCACAACAGTCAATGGCTAATTGGCCTCCATCGTTCACTGGTTGACATTTGCGATCGGCCCAACACT \\
\hline TF & Myb3R1 & XM_002182738.1 & Phatr3_J15016 & GTATGATCCTGGAGTGTCATTTGACGTTGGGAAACCGCTGGGCCGAAATTGCCAAGCGTCTACCGGGAAGAACTGATAACGCTATTAAAAATCACTGGAA \\
\hline TF & Myb3R2 & XM_002180449.1 & Phatr3_J6839 & CGAATTCTCATAGGAGCACAGGACACAATGGGAAATCGATGGGCTGAGATTGCCAAGCGGCTGCCAGGACGTACGGACAATGCGATAAAGAATCGCTGGA \\
\hline TF & Myb3R4 & XM_002180741.1 & Phatr3_J36337 & GACAGATGCCGAAGACGCAATTGTAATGGATGCGGTCAATTCCAGCTCGGAGCAACCATTTACTCGTTGGTCAGACTTGGCGCAACGTCTACCCGGACGT \\
\hline TF & Myb3R5 & XM_002184658.1 & Phatr3_J7959 & GACGTCGTTATCTGAATTGCTTGGACCCAACAATCCGAAAAGACGAATGGACGGAGCAAGAGAAAGAAGCTTTGTTTGATGCACAGCGGCGCATGGGAAA \\
\hline TF & Myb5R & XM_002185245.1 & Phatr3_J50365 & TCAACACCACATAAACGCTCGTTTCAGGCTTTGGATGTGTTAGTGAACGCGGTTGGTTT'TCATCGAGAACCAAATGACCAAATGAAGCAGACGAAGATGT \\
\hline Cell cycle & PCNA & XM_002182225.1 & Phatr3_J29196 & AATGCAGGAACCGGTCGAACTCACCTTTGCCCTGCGCTACCTCAACTTTTTTACCAAGGCGACTCCGTTGAGTGGACACGTCATTATCTCCATGGCACCG \\
\hline Metabolism & Pds1 & XM_002180135.1 & Phatr3_J35509 & AGCATGGCCAAGGCACTCGATTTCATTGATCCCGACAAATTGAGTATGACGGTCGTTTTGACCGCCATGAATCGCTTTTTGAACGAAGACAACGGCCTCC \\
\hline Metabolism & petA & GI:118411009 & \#/A & TCTCTGGATCAGGAGCCATAACAGGGAAAATTAACTCTTGATGAGTCTTTCCTGCAATTGGTCCAACTACTAAGATATTATCAAATTCACTACTATACGG \\
\hline Metabolism & Pgr5 & XM_002178672.1 & Phatr3_J44748 & GTAACGTTCGCGCTTCTGATCGCTACCGCTGCCGCGTTTCGCGTGGCGGGCGGAGCCACCGGGGTTCTTCCTTTGTGCTTTCCCGACGAACCAGCATCG \\
\hline Metabolism & PgrL & XM_002177043.1 & Phatr3_J42543 & CCACAACAAGTAGCGTTTCCAATTTAGACAAGACTACGAACAAACGATGCGAGTCAACTCTGTGGTATTTGGCCTTTTGGTGTTCTCGGCCGTCAGCGAT \\
\hline Metabolism & Por1 & XM_002179653.1 & Phatr3_J12155 & TCGGGTGGTGAGATTTTTGAGAATCAGCAGTCCGACGCAGTACGGGATCTTCCTACCGCCAAGAAAATGTGGAAACTGAGTAGGGAAGCAGTTGGTCTTT \\
\hline Metabolism & Por2 & XM_002180956.1 & Phatr3_J13001 & AATCACCACTATTCCGTGAAAAGCGCCCGTGGTTCCGTAAATACTTTCCCATCTTTATGAAGTTTATTACGGGAGGATACGTTGGAGAGCACGAAGCCGG \\
\hline Metabolism & Por3 & XM_002177432.1 & Phatr3_J43164 & CGCGTTCTCAACACGAGGAAAAGCGGTGAGACTCATGATTCGTGATCCGACCAGTAGTCGTATCGAGGCACACCGTAGCGTGGGAGACGTAGACGTTCCC \\
\hline Metabolism & psaA & GI:118410964 & \#N/A & GCTAAGAAGAACGCCCAAGTAGTTCCAATACCACCCAATAAATAATGAGCTAAACCAACTGCACGACCCTGAGTAATGCTTAATGCACGAGGTTGGATTG \\
\hline Metabolism & psaD & Gl:118410999 & \#N/A & TTAAATGTACGTAATTGGGTTCCCAATGCTAAACATTGCTCTTTGCGCGCTAAGTATAATAAATTTTCTCCACTACG \\
\hline
\end{tabular}




\begin{tabular}{|c|c|c|c|c|}
\hline Metabolism & $\mathrm{psbc}$ & HQ912250.1 & $\# \mathrm{~N} / \mathrm{A}$ & GGCCCAACAGGTCCTGAGGCTTCACAAGCACAAGCATTCACGTT'TTAGTTCGAGATCAACGTTTAGGTGCTAATGTTTCATCAGCACAGGGTCCAACTG \\
\hline Metabolism & Psy1 & XM_002178740.1 & Phatr3_EG02349 & ACCTGTCGTCGTGGGAATTGCGATTGGAGCGTTTGTGGCAATACGGACAAGTACAGGATGTCTTTGACCTGTGCTTGCTAGATCTTCGAATCCAGTATCC \\
\hline Reference Gene & Rps & XM_002178225.1 & Phatr3_J10847 & CTCCGCGCATTTTTGCCCGGTTCCCATTTGACCGGACAGTTGCCCGACGAAGATCTCATTGGAAACAACTTGCAGCTTAAATTCCTCGAAGTCAACCAGG \\
\hline TF & Sigma70.1a & XM_002182291.1 & Phatr3_J14599 & GACAATTAGGGGTATCTCGGGATCGGATTCGCCTTGTCGAAACCCGAGCACTGAACAAATTGCGACACCCGCAACAAAATTACAAGCTGCAGTCTTACGT \\
\hline TF & Sigma70.1b & XM_002182124.1 & Phatr3_J3388 & TTCTTGGTCATTGGAAGAAACGGCCAAACAACTAGGGATGTCTCGGGATCGGATTCGCCTTGTCGAAGCCCGAGCACTGAATAAATTGCGACGTCCACAA \\
\hline TF & Sigma70.2 & XM_002178682.1 & Phatr3_J5537 & ACGTGGATTGCGATTCGCCACCTACGCCACTTACTGGGTCACCAATTCCGTACGCTTCTGCTTCCAAACCGCATCCACCGGATGTTTGGCGGGTACCGGTA \\
\hline TF & Sigma70.3 & XM_002185461.1 & Phatr3_J17029 & TATGATGGTTCCTGATCTAAAGAAGCAAATTCGTCGCTCGCTTCGCGCCAAGGCTGCCCTGACGGAATCAAATATTCGACTCGTGGTATCCATTGCGAAA \\
\hline TF & Sigma70.4 & XM_002177232.1 & Phatr3_J9312 & AACCCGAAAGATCTCGGTCACGAAAGTCACGATTTGCTAGGGGACACAATCTCCGCCAGTAGTGCTCTTTTTGACGAAAGCACACCCGAAAAACGAGTCG \\
\hline TF & Sigma70.5 & XM_002182854.1 & Phatr__J14908 & AAGAACTCATACAGGAAGGCAGTTTGGGATTGTTGCGCGCGGCCGAATTGTTTGATCCCGAGCGAGGACTGCGATTCAGCACTTACGCGGTAGTGTGGAT \\
\hline TF & Sigma70.6 & XM_002178042.1 & Phatr3_J9855 & CTGGAAAAATCAATATGGAAGCTATTCGGCAGACCATTGAAGAAGGACTGGAAGCCAAAAATCAGCTAGTCACTTCGAATCTGCGGATGGTACAGAGTGT \\
\hline TF & Sigma70.7 & XM_002185047.1 & Phatr__J50183 & AATGATGACGCTCCTCTCGCATACGGTGGAACTCCGTCGTATCAAGCAAATCGAAGCTGATCTTGCTTTGACGTATCCATCTTCCCGGAAACCCACTACT \\
\hline TF & Taz2 & XM_002178935.1 & Phatr3_J44807 & CCTAATGTAGAGACTGTAGTGGTAAAAGCGAAGCGCGCAGCCTCGACTATTTGGACTTTACTTCATGCACAGAATTGCGTTTTGGGGGACAGATGCCCAC \\
\hline Reference Gene & Tbp & XM_002186285.1 & Phatr__10199 & CTGATTTTTTCCAGCGGTAAAATGTGCGTGACCGGAGTCAAGAGCACACACAACGCCAATCTGGCGGCGAAAAAGTTTGCCTACATTGTGGAGCGCGTCG \\
\hline Metabolism & Vde & XM_002178607.1 & Phatr__J51703 & GGCGATGACGGATCATATCCCGTTCCCGCACCCGAAATCGTCGTACCCAAGTTTGACACCAAGTTCTTCGATGGACGACTCTACATCTCAGCCGGACAAA \\
\hline Metabolism & Vdl1 & XM_002180599.1 & Phatr__J36048 & AAGGCTCTCTTTAGCAATCCGCGTGGAATCAAAGGTGTCTCCTGTTTGGGACGCTGCAAGGGCGAGCAATCGTGTGCGACGCGGTGTTTTGCCGAATTCG \\
\hline Metabolism & Vdl2 & XM_002180015.1 & Phatr3_445846 & ACACACCGCCTTCGGCAATGAATATCCAATTTCCGGAGCCAGACGAATCACTCCATGTCTGGGACCACGTACGAAACATCGGTAAAGTGTGTACCGGGTT \\
\hline Metabolism & Vdr & XM_002177477.1 & Phatr3_J43240 & GTCGCTTGCGGTGCCAACGAAGCCTATGATAAGTTTCCTTCGCAGAACCAACTCTTCTATCCCGCTGCTCGCGGTCGAGATTTGTGGTACGATCCCGTTT \\
\hline Metabolism & Zds & XM_002184417.1 & Phatr3_J30514 & CGGCGTCAACGAATCCATGCGGGACACCGCCAACGTACTCGCCCGTTTTAATTCACTCATTCTCGCCCGAGTCT'TTGGACACGCCGACGTCACGGAACTC \\
\hline Metabolism & Zep1 & XM_002179586.1 & Phatr__J45485 & TTCCCAATGAAGTTTTCTACCACGGTGTCATCGGCACTGTTCCTGATCGCGTCGGTATCGACCACGACTTCCTTCACACCCGTCCAGTCCTTTGGCGTGC \\
\hline Metabolism & Zep3 & XM_002178331.1 & Phatr3_J10970 & CCAAGGTTTTGACCAAGATGCCCACCATGGATGTTACCGTACTGGAACAAACGTCCGAATTCAAACGGTTCGGTGGACCCATCCAGCTCGCTAGTAACGC \\
\hline & & & & \\
\hline
\end{tabular}

823

824

825 Table S2: Calculated phases, amplitudes and periods of the $\mathrm{Wt}$ and $\mathrm{PtbHlH1a}$ OE lines growing 826 in 16L:8D. (P., Period; Ph., phase; Amp., amplitude; Std, standard deviation; t-test, t-test P827 value between $\mathrm{Wt}$ and the indicated line).

\begin{tabular}{|l|c|c|c|l|c|c|c|c|c|l|}
\hline Data & N & Period & P.Std & P. t-test & Phase & Ph.Std & Ph. t-test & Amp. & Amp.Std & Amp.t-test \\
\hline Wt & 8 & 23.7 & 0.32 & & 13.02 & 0.54 & & $8.30 \mathrm{E}-01$ & $1.20 \mathrm{E}-01$ & \\
\hline OE-1 & 5 & 23.37 & 0.51 & 0.168894675 & 15.93 & 1.83 & 0.001213017 & $5.60 \mathrm{E}-01$ & $2.60 \mathrm{E}-01$ & 0.02447126 \\
\hline OE-2 & 6 & 23.38 & 0.48 & 0.159824505 & 15.19 & 1.52 & 0.002642208 & $6.10 \mathrm{E}-01$ & $7.00 \mathrm{E}-02$ & 0.001606661 \\
\hline OE-3 & 4 & 23.89 & 0.22 & 0.323356278 & 13.94 & 0.51 & 0.017556508 & $8.40 \mathrm{E}-01$ & $1.20 \mathrm{E}-01$ & 0.841165049 \\
\hline
\end{tabular}

828

829

830 Table S3: Accession numbers of the proteins utilized in the bHLH-PAS phylogenetic analysis.

\begin{tabular}{|l|l|l|l|}
\hline \multicolumn{1}{|c|}{ SPECIES } & ABBREVIATION & & ACCESSION \\
\hline Amphiprora sp., Strain CCMP467 & Amph & CAMPEP_0168730192 & \\
\hline Asterionellopsis glacialis, Strain CCMP134 & Agla2 & CAMPEP 0195290872 & \\
\hline Asterionellopsis glacialis, Strain CCMP1581 & Agla3 & CAMPEP_0197142484 & \\
\hline Astrosyne radiata, Strain 13vi08-1A & Arad1 & CAMPEP_0116831938 & \\
\hline
\end{tabular}


bioRxiv preprint doi: https://doi.org/10.1101/271445; this version posted May 26, 2018. The copyright holder for this preprint (which was not certified by peer review) is the author/funder. All rights reserved. No reuse allowed without permission.

\begin{tabular}{|c|c|c|c|}
\hline Astrosyne radiata, Strain $13 \mathrm{vi} 08-1 \mathrm{~A}$ & Arad2 & CAMPEP_0116837632 & \\
\hline Attheya septentrionalis, Strain CCMP 2084 & Asep & CAMPEP_0198303966 & \\
\hline Capsaspora owczarzaki ATCC 30864 & Cowc1 & XP 004345696 & \\
\hline Capsaspora owczarzaki ATCC 30864 & Cowc2 & $X P \_004343694$ & \\
\hline Chaetoceros debilis, Strain MM31A-1 & Cdeb & CAMPEP 0194099558 & \\
\hline Chaetoceros dichaeta, Strain CCMP1751 & Cdic & CAMPEP_0198277646 & \\
\hline Chaetoceros neogracile, Strain CCMP1317 & Cneo2 & CAMPEP 0195415676 & \\
\hline Chaetoceros neogracile, Strain CCMP1317 & Cneo3 & CAMPEP_0195453836 & \\
\hline Chaetoceros sp., Strain GSL56 & Chae & CAMPEP_0176495412 & \\
\hline Corethron hystrix, Strain 308 & Chys & CAMPEP 0113330172 & \\
\hline Corethron pennatum, Strain L29A3 & Cpen & CAMPEP_0194280324 & \\
\hline Cyclophora tenuis, Strain ECT3854 & Cten 1 & CAMPEP 0116540656 & \\
\hline Cyclophora tenuis, Strain ECT3854 & Cten2 & CAMPEP_0116579710 & \\
\hline Cyclotella meneghiniana, Strain CCMP 338 & Cmen & CAMPEP 0172279412 & \\
\hline Cylindrotheca closterium & Cclo & CAMPEP_0113624952 & \\
\hline Dactyliosolen fragilissimus & Dfra & CAMPEP_0184871870 & \\
\hline Detonula confervacea, Strain CCMP 353 & Dcon & CAMPEP_0172306268 & \\
\hline Ditylum brightwellii, Strain Pop2 (SS10) & Dbri & CAMPEP_0181012646 & \\
\hline Drosophila melanogaster & $\mathrm{dmClock}$ & AAC62234 & FBpp0099478 \\
\hline Drosophila melanogaster & dmCycle & NP_ 524168 & FBpp00 074693 \\
\hline Drosophila melanogaster & dmSim & AAC64519 & FBpp00 02178 \\
\hline Drosophila melanogaster & dmTango & NP_ 731308 & FBpp00 081483 \\
\hline Drosophila melanogaster & dmTrh & AAA96754 & FBpp0293065 \\
\hline Durinskia baltica, Strain CSIRO CS-38 & Dbal & CAMPEP 0170381008 & \\
\hline Ectocarpus siliculosus & Esill & Ec-02_004780 PAS domain (681) ;mRNA; f:5082087-5086119 & \\
\hline Eucampia antarctica, Strain CCMP 1452 & Eant & CAMPEP 0197830682 & \\
\hline Extubocellulus spinifer, Strain CCMP396 & Espi1 & CAMPEP_0178587872 & \\
\hline Extubocellulus spinifer, Strain CCMP396 & Espi2 & CAMPEP_0178723458 & \\
\hline Fragilariopsis kerguelensis, Strain L26-C5 & Fkue1 & CAMPEP_0170867208 & \\
\hline Fragilariopsis kerguelensis, Strain L26-C5 & Fkue2 & CAMPEP_0170907188 & \\
\hline Fragylariopsis cylindrus & FcbHLH1a & jgi|Fracy1|204663 & \\
\hline Fragylariopsis cylindrus & FcbHLH4 & jgi|Fracy1|241747 & \\
\hline Fragylariopsis cylindrus & FCbHLH7 & jgi|Fracy1|172104 & \\
\hline Galdieria sulphuraria & Gsul & XP_005709404 & \\
\hline Glenodinium foliaceum, Strain CCAP $1116 / 3$ & Gfoll & CAMPEP_0167841300 & \\
\hline Grammatophora oceanica, Strain CCMP 410 & Goce & CAMPEP 0194036250 & \\
\hline Guillardia theta CCMP2712 & Gthe1 & $X P \_005835918$ & \\
\hline Homo sapiens & hsArnt & AAA51777 & \\
\hline Homo sapiens & hsbMal1 & NP_1284651 & \\
\hline Homo sapiens & hsClock & AAB83969 & \\
\hline Homo sapiens & hsNpas1 & AAH 39016 & \\
\hline Homo sapiens & hsSim2 & NP_5060 & \\
\hline $\begin{array}{l}\text { Kryptoperidinium foliaceum, Strain CCMP } \\
1326\end{array}$ & Kfoll & CAMPEP_0176099000 & \\
\hline
\end{tabular}


bioRxiv preprint doi: https://doi.org/10.1101/271445; this version posted May 26, 2018. The copyright holder for this preprint (which was not certified by peer review) is the author/funder. All rights reserved. No reuse allowed without permission.

\begin{tabular}{|c|c|c|c|}
\hline $\begin{array}{l}\text { Leptocylindrus danicus var. danicus, Strain } \\
\text { B650 }\end{array}$ & $\operatorname{Ldan} 1$ & CAMPEP_0116039394 & \\
\hline $\begin{array}{l}\text { Leptocylindrus danicus var. danicus, Strain } \\
\text { B651 }\end{array}$ & Ldan2 & CAMPEP_0116053596 & \\
\hline Leptocylindrus danicus, Strain CCMP1856 & Ldan 3 & CAMPEP 0196809694 & \\
\hline Licmophora paradoxa, Strain CCMP2313 & Lpar & CAMPEP_0202478474 & \\
\hline Minutocellus polymorphus, Strain RCC2270 & Mpol & CAMPEP 0185804732 & \\
\hline Monosiga brevicollis & Mbre2 & jgi|Monbr1|26507| & \\
\hline Mus musculus & mmArnt & AAA56717 & \\
\hline Mus musculus & mmbMal1b & BAD26600 & \\
\hline Mus musculus & mmbMal2 & $\mathrm{ABC} 50103$ & \\
\hline Mus musculus & mmClock & AAC53200 & \\
\hline Mus musculus & mmNpas1 & NP_ 32744 & \\
\hline Mus musculus & mmSim2 & AAA91202 & \\
\hline Nannochloropsis gaditana CCMP526 & Ngad_2 & Naga_100016g14 & \\
\hline Nannochloropsis gaditana CCMP526 & Ngad 3 & Naga $100165 \mathrm{~g} 4$ & \\
\hline Nannochloropsis oceanica CCMP1779 & Noce & NannoCCMP1779_9983-mRNA-1 & \\
\hline Nannochloropsis oceanica CCMP1779 & Noce_2 & NannoCCMP1779_1600-mRNA-1 & \\
\hline Nitzschia punctata, Strain CCMP561 & Npun & CAMPEP_0178827522 & \\
\hline Nitzschia sp. & Nitz & CAMPEP_0113465330 & \\
\hline Phaeodactylum tricornutum & PtbHLHla & jgi|Phatr2|44962 & Phatr3 J44962 \\
\hline Phaeodactylum tricornutum & PtbHLH1b & jgi|Phatr2| 44963 & Phatr3_J44963 \\
\hline Phaeodactylum tricornutum & PtbHLH2 & jgi|Phatr2|54435 & Phatr3 J54435 \\
\hline Proboscia alata, strain PI-D3 & $\mathrm{Pala}$ & CAMPEP_0194393728 & \\
\hline Pseudo-nitzschia arenysensis, Strain B593 & Pare & CAMPEP_0116139930 & \\
\hline $\begin{array}{l}\text { Pseudo-nitzschia australis, Strain } 1024910 \\
A B\end{array}$ & Paus 1 & CAMPEP 0168282354 & \\
\hline $\begin{array}{l}\text { Pseudo-nitzschia australis, Strain } 1024910 \\
\text { AB }\end{array}$ & Paus2 & CAMPEP_0168310394 & \\
\hline Pseudo-nitzschia delicatissima, Strain B596 & Pdel1 & CAMPEP 0116091770 & \\
\hline Pseudo-nitzschia delicatissima, Strain B596 & Pdel2 & CAMPEP_0116094064 & \\
\hline Pseudo-nitzschia fraudulenta, Strain WWA7 & Pfral & CAMPEP_0201229664 & \\
\hline Pseudo-nitzschia fraudulenta, Strain WWA7 & Pfra2 & CAMPEP_0201232896 & \\
\hline Pseudo-nitzschia heimii, Strain UNC1101 & Pheil & CAMPEP_0197189528 & \\
\hline Pseudo-nitzschia heimii, Strain UNC1101 & Phei2 & CAMPEP 0197199168 & \\
\hline Pseudo-nitzschia multiseries & PmbHLH1a & jgi|Psemu1|26622 & \\
\hline Pseudo-nitzschia multiseries & PmbHLH7 & jgi|Psemu1|228145 & \\
\hline $\begin{array}{l}\text { Pseudo-nitzschia pungens, Strain cf. } \\
\text { pungens }\end{array}$ & Ppun & CAMPEP_0172413298 & \\
\hline Rhizosolenia setigera, Strain CCMP 1694 & Rset & CAMPEP_0178942232 & \\
\hline Skeletonema costatum, Strain 1716 & $\mathrm{~S} \cos 1$ & CAMPEP 0113408658 & \\
\hline Skeletonema dohrnii, Strain SkelB & Sdoh & CAMPEP_0195221452 & \\
\hline Skeletonema japonicum, Strain CCMP2506 & Sjap & CAMPEP 0201725346 & \\
\hline Skeletonema marinoi, Strain FE7 & Smar1 & CAMPEP_0180846198 & \\
\hline Skeletonema marinoi, Strain UNC1201 & Smar2 & CAMPEP 0197231700 & \\
\hline Skeletonema menzelii, Strain CCMP793 & Smen & CAMPEP_0183679140 & \\
\hline Stauroneis constricta, Strain CCMP 1120 & Scon 1 & CAMPEP_0119548954 & \\
\hline Stauroneis constricta, Strain CCMP1120 & Scon2 & CAMPEP_0119572288 & \\
\hline
\end{tabular}


bioRxiv preprint doi: https://doi.org/10.1101/271445; this version posted May 26, 2018. The copyright holder for this preprint (which was not certified by peer review) is the author/funder. All rights reserved. No reuse allowed without permission.

\begin{tabular}{|c|c|c|}
\hline Staurosira complex sp., Strain CCMP2646 & Stau & CAMPEP_0202487246 \\
\hline Synedropsis recta cf, Strain CCMP 1620 & Srec1 & CAMPEP_0119009808 \\
\hline Synedropsis recta cf, Strain CCMP1620 & Srec 2 & CAMPEP 0119029558 \\
\hline $\begin{array}{l}\text { Thalassionema frauenfeldii, Strain CCMP } \\
1798\end{array}$ & Tfral & CAMPEP_0178899126 \\
\hline $\begin{array}{l}\text { Thalassionema frauenfeldii, Strain CCMP } \\
1798\end{array}$ & Tfra2 & CAMPEP 0178923098 \\
\hline Thalassionema nitzschioides, Strain L26-B & Tnit1 & CAMPEP_0194218124 \\
\hline Thalassionema nitzschioides, strain L26-B & Tnit2 & CAMPEP 0194240022 \\
\hline Thalassiosira antarctica, Strain CCMP 982 & Tant & CAMPEP_0202006238 \\
\hline Thalassiosira gravida, Strain GMp14c1 & Tgra & CAMPEP_0201684800 \\
\hline Thalassiosira miniscula, Strain CCMP1093 & $\operatorname{Tmin}$ & CAMPEP 0183747574 \\
\hline Thalassiosira oceanica & Toce 2 & EJK 65393 \\
\hline Thalassiosira pseudonana & TpbHLH1 & jgi|Thaps 3124007 \\
\hline Thalassiosira pseudonana & ТрьнLн2 & jgi|Thaps3|20899 \\
\hline Thalassiosira pseudonana & TрьнLн7 & jgi|Thaps $3 \mid 23208$ \\
\hline Thalassiosira sp., Strain FW & $\mathrm{Tfw}$ & CAMPEP_0172354094 \\
\hline Thalassiosira weissflogii, Strain CCMP1010 & Twei & CAMPEP_0203515806 \\
\hline Thalassiothrix antarctica, Strain L6-D1 & Txnt & CAMPEP_0194143228 \\
\hline Tiarina fusus, Strain LIS & Tfus 1 & CAMPEP_0117003500 \\
\hline Tiarina fusus, Strain LIS & Tfus 3 & CAMPEP_0117046192 \\
\hline
\end{tabular}

831

832 
833 Table S4: List of the oligonucleotides used in this work.

\begin{tabular}{|c|c|c|c|c|}
\hline Gene & Phatr3 Accession & Oligo name & Sequence & Type \\
\hline \multirow{6}{*}{$b H L H 1 a$} & \multirow{6}{*}{ Phatr3_J44962 } & bHLH1a-endogenous-QFw & TTATGTCTTTCGGCGACGGG & \multirow{2}{*}{ QPCR } \\
\hline & & bHLH1a-endogenous-QRv & AGCAACGAATGCATGCAAGG & \\
\hline & & bHLH1a-total-QFw & ATTCTTGGTCCCACCCGGTA & \multirow{2}{*}{ QPCR } \\
\hline & & bHLH1a-total-QRv & ACGCCACATTGAAAAACCGAG & \\
\hline & & Pt-bHLH1a-Dral-Fw & GATTTTAAAATGAATAAGCCAGGACAGCG & \multirow{2}{*}{ Full lenght cloning } \\
\hline & & Pt-bHLH1a-Xhol-Rv & TTGCTCGAGCACAGCTTCGCTGCATCGTC & \\
\hline \multirow{2}{*}{$b H L H 1 b$} & \multirow{2}{*}{ Phatr3_J44963 } & bHLH1b-QFw & TGCGATCTCAACGGCTAATA & \multirow{2}{*}{ QPCR } \\
\hline & & bHLH1b-QRv & CGCAAAACGAGGCTAATTTC & \\
\hline \multirow{2}{*}{ bHLH3 } & \multirow{2}{*}{ Phatr3_J42586 } & bHLH3-QFw & САCTCTCATCATGCGGGAAT & \multirow{2}{*}{ QPCR } \\
\hline & & bHLH3-QRv & GCGCGTTGTCTTCCTCTATC & \\
\hline \multirow{2}{*}{$b Z I P 7$} & \multirow{2}{*}{ Phatr3_J48800 } & bZIP7-QFw & CCTTATTGATATTCAAGATTCCAAGG & \multirow{2}{*}{ QPCR } \\
\hline & & bZIP7-QRv & GTTTCGGAACCTGCATAGGA & \\
\hline \multirow{2}{*}{ CDKA1 } & \multirow{2}{*}{ Phatr3_J20262 } & CDKA1-QFw & AGCGGTATCAAAGGATGGAAAAG & \multirow{2}{*}{ QPCR } \\
\hline & & CDKA1-QRv & CTTCATCTTCGGCTTCAAGGC & \\
\hline \multirow{2}{*}{$C D K D$} & \multirow{2}{*}{ Phatr3_J10160 } & CDKD-QFw & АTTACTTCTGCGGAGACCATTC & \multirow{2}{*}{ QPCR } \\
\hline & & CDKD-QRv & GCGGTAAAGATTTCGTCAAAGG & \\
\hline \multirow{2}{*}{$C Y C A / B ; 1$} & \multirow{2}{*}{ Phatr3_J17135 } & CYCA/B1-QFw & TACACCGCCACTCCAAGAC & \multirow{2}{*}{ QPCR } \\
\hline & & CYCA/B1-QRv & TCGGAGGACGGGATGGG & \\
\hline \multirow{2}{*}{ CYCB1 } & \multirow{2}{*}{ Phatr3_J46095 } & CYCB1-QFw & TCCTGGTCCGCTACTTGAAAG & \multirow{2}{*}{ QPCR } \\
\hline & & CYCB1-QRv & GCTGGCTGGGAAGATAACGC & \\
\hline \multirow{2}{*}{ Por2 } & \multirow{2}{*}{ Phatr3_J13001 } & Por2-QFw & CCTGGTTGCATTGCCGAATC & \multirow{2}{*}{ QPCR } \\
\hline & & Por2-QRv & TCTCCAACGTATCCTCCCGT & \\
\hline \multirow{2}{*}{ Rps } & \multirow{2}{*}{ Phatr3_J10847 } & Rps-QFw & CGAAGTCAACCAGGAAACCAA & \multirow{2}{*}{ QPCR } \\
\hline & & Rps-QRv & GTGCAAGAGACCGGACATACC & \\
\hline Thn & Phatr3 110199 & Tbp-QFw & ACCGGAGTCAAGAGCACACAC & $O P C P$ \\
\hline & & Tbp-QRv & CGGAATGCGCGTATACCAGT & \\
\hline$V D R$ & Phatr3 143240 & VDR-QFw & TTTCCTTCGCAGAACCAACT & OPCR \\
\hline & & VDR-QRv & TTGTCGAGCACTGAAAATCG & \\
\hline
\end{tabular}

834

835

836

837

838

839

840

841 


\section{$842 \quad$ References}

843 1. Zielinski T, Moore AM, Troup E, Halliday KJ, \& Millar AJ (2014) Strengths and limitations $844 \quad$ of period estimation methods for circadian data. PLoS One 9(5):e96462.

845 2. Agier N \& Fischer G (2016) A Versatile Procedure to Generate Genome-Wide Spatiotemporal Program of Replication in Yeast Species. Methods Mol Biol 1361:247-264 .

3. Geiss GK, et al. (2008) Direct multiplexed measurement of gene expression with color-coded probe pairs. Nature biotechnology 26(3):317-325.

4. Chauton MS, Winge P, Brembu T, Vadstein O, \& Bones AM (2013) Gene regulation of carbon fixation, storage, and utilization in the diatom Phaeodactylum tricornutum acclimated to light/dark cycles. Plant physiology 161(2):1034-1048.

5. Saeed AI, et al. (2003) TM4: a free, open-source system for microarray data management and analysis. Biotechniques 34(2):374-378.

6. Siaut M, et al. (2007) Molecular toolbox for studying diatom biology in Phaeodactylum tricornutum. Gene 406(1-2):23-35.

7. Keeling PJ, et al. (2014) The Marine Microbial Eukaryote Transcriptome Sequencing Project (MMETSP): Illuminating the Functional Diversity of Eukaryotic Life in the Oceans through Transcriptome Sequencing. Plos Biol 12(6).

8. Katoh K \& Standley DM (2013) MAFFT multiple sequence alignment software version 7: improvements in performance and usability. Mol Biol Evol 30(4):772-780.

9. Kumar S, Stecher G, \& Tamura K (2016) MEGA7: Molecular Evolutionary Genetics Analysis Version 7.0 for Bigger Datasets. Mol Biol Evol 33(7):1870-1874.

10. Darriba D, Taboada GL, Doallo R, \& Posada D (2011) ProtTest 3: fast selection of best-fit models of protein evolution. Bioinformatics 27(8):1164-1165.

11. Miller MA, Pfeiffer, W., and Schwartz, T. (2010) Creating the CIPRES Science Gateway for Inference of Large PhylogeneticTrees. In Proceedings of the Gateway Computing Environments Workshop (GCE) (New Orleans, LA):1-8. 